\title{
STABLE REDUCTIVE VARIETIES I: AFFINE VARIETIES
}

\author{
VALERY ALEXEEV AND MICHEL BRION
}

\section{Contents}

0. Introduction 1

1. Main definitions and results 3

2. General criteria 6

2.1. Seminormality and connectedness of isotropy groups 6

2.2. Finiteness of number of orbits and group-like condition 9

3. Orbits in stable reductive varieties 11

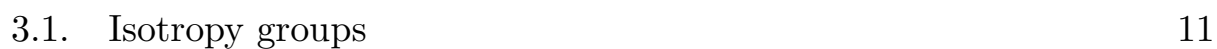

3.2. Algebras of regular functions 14

$\begin{array}{ll}\text { 4. Reductive varieties } & 18\end{array}$

$\begin{array}{ll}\text { 4.1. Classification } & 18\end{array}$

4.2. Associated stable toric varieties 20

5. Stable reductive varieties 24

6. Self-adioint stable reductive semigroups 26

7. Families 32

7.1. General remarks 32

7.2. Moduli of embedded stable reductive varieties 35

$\begin{array}{ll}\text { 7.3. Local isotrivialitv } & 38\end{array}$

7.4. One-parameter degenerations 39

7.5. The Vinberg familv 43

7.6. Local structure of families of reductive varieties 45

$\begin{array}{ll}\text { References } & 46\end{array}$

\section{INTRODUCTION}

The main motivation for this work is to extend the construction of the moduli spaces of polarized abelian varieties $A_{g}$ and their compactifications to a non-commutative setting. Recall that $A_{g}$ admits many natural compactifications: toroidal (due to Mumford et al), Satake-Baily-Borel, Borel-Serre etc. Of these, one special toroidal compactification has an interpretation as the moduli space of projective stable pairs $(X, D)$ : see Ale02, which extends the much earlier work of Namikawa Nam76]. The former paper also

Date: September 2, 2003. 
constructs the moduli space of stable pairs with torus action, and semiabelian action.

This raises the question of defining a nice class of varieties with an action of a given non-commutative algebraic group $G$, which includes the group itself, its equivariant compactifications, and also all of their natural degenerations, "stable" varieties. In addition, one would like to construct a nice, complete moduli space of these stable varieties, so to say, a toroidal compactification.

That is exactly what we do here, for a connected reductive group $G$. It comes equipped with two commuting $G$-actions by left and right multiplication, that is, with an action of $G \times G$; this reduces to a $G$-action if $G$ is commutative. We define a class of reductive varieties, consisting of (irreducible) varieties with a $G \times G$-action. It contains the group $G$ and all of its equivariant embeddings, and is closed under (flat) degenerations with reduced and irreducible fibers. We also introduce a larger class of (possibly reducible) stable reductive varieties, closed under degenerations with reduced fibers.

In the commutative case, where $G$ is a torus, reductive varieties are just toric varieties; in general, they are certain, highly symmetric, spherical varieties, and stable reductive varieties are obtained from them by a simple glueing procedure.

In the present paper, we concentrate on affine stable reductive varieties, the projective case being the topic of our subsequent paper SRV-II]. We classify them all in terms of combinatorial data; these turn out to be complexes of cones in the weight space of $G$, invariant under the action of the Weyl group $W$. We describe all their one-parameter degenerations. Like in the toric case, they arise from certain stable reductive varieties for the larger group $\mathbb{G}_{m} \times G$.

An interesting consequence of the classification is the "toric correspondence": it turns out that stable reductive varieties are in bijection with stable toric varieties equipped with a compatible $W$-action.

Another nice feature of reductive varieties is the existence of an associative multiplication law, making each of them into an algebraic semigroup (generally without identity element). We characterize the class of semigroups arising in this way, that we call reductive semigroups. It contains the class of (normal) reductive monoids studied by Putcha, Renner and Rittatore (see [Rit98] and the survey [Sol99]), that is, of algebraic semigroups with an identity element and a reductive unit group.

Finally, we begin the study of families of stable reductive varieties and, more generally, of varieties with reductive group action. Examples of these already occur in work of Popov Pop86 constructing a special degeneration of any affine $G$-variety, and of Vinberg Vin95 on a remarkable family of reductive monoids. Given a reductive variety $X$, we construct a family à la Vinberg of such varieties, with general fiber $X$, and we prove that any (reduced and irreducible) degeneration of $X$ arises in that way. 
We also construct a fine moduli space for families of $G$-subvarieties of a fixed $G$-module. The construction applies to all multiplicity-finite $G$ varieties (not only to stable reductive varieties), and is an application and a generalization of the multigraded Hilbert scheme of Haiman and Sturmfels HS02. This result plays a fundamental rôle in our follow-up paper SRV-II; there we construct the moduli space of projective pairs $(X, D)$ analogous to the Deligne-Mumford-Knudsen moduli space of stable curves, and explore connections with log Minimal Model Program.

\section{MAin DEFInitions AND RESUlts}

We will use Har77] as a general reference for algebraic geometry, and PopVin94, Gro97] for algebraic transformation groups.

Notation 1.1. (on groups).

The ground field $k$ is algebraically closed, of characteristic zero. Let $G$ be a connected reductive group over $k$. Let $B, B^{-}$be opposite Borel subgroups of $G$, with unipotent radicals $U, U^{-}$. Then $T=B \cap B^{-}$is a maximal torus of $G$; let $N=N_{G}(T)$ be its normalizer, and $W=N_{G}(T) / T$ the corresponding Weyl group. There exists a unique automorphism $\theta$ of $G$ such that $\theta(t)=t^{-1}$ for any $t \in T$. Then $\theta$ is involutive; it exchanges $B$ with $B^{-}$, stabilizes $N$ and $T$, and fixes pointwise $W$.

The product $G \times G$ is a connected reductive group; the subgroups $B^{-} \times B$, $B \times B^{-}$are opposite Borel subgroups, with common torus $T \times T$. The map $\Theta:\left(g_{1}, g_{2}\right) \mapsto\left(\theta\left(g_{2}\right), \theta\left(g_{1}\right)\right)$ is an involutive automorphism of $G \times G$ (note the additional twist). The diagonal subgroups $\operatorname{diag} G, \operatorname{diag} N, \operatorname{diag} T$ are $\Theta$-invariant, and the induced map on $\operatorname{diag} W$ is the identity.

Notation 1.2. (on varieties). By a variety, we mean a reduced scheme of finite type over $k$; in particular, varieties need not be irreducible. A $G$ variety $X$ is a variety endowed with an action of $G$. The group of equivariant automorphisms of $X$ is then denoted by $\operatorname{Aut}^{G}(X)$. As an example, for any homogeneous space $G / H$, where $H$ is a closed subgroup of $G$, we have $\operatorname{Aut}^{G}(G / H)=N_{G}(H) / H$. The fixed point subset of $H$ in $X$, regarded as a closed reduced subscheme, is denoted by $X^{H}$; it has an action of $N_{G}(H) / H$. The stabilizer of a closed subset $Y \subseteq X$ is the closed subgroup $\operatorname{Stab}_{G}(Y)=$ $\{g \in G \mid g Y=Y\}$ of $G$.

We will mostly consider $G \times G$-varieties. The adjoint of such a variety $X$ is the $G \times G$-variety $\Theta(X)$ with underlying variety $X$, and action twisted by $\Theta$. (If $X$ is a $G$-variety, we define another $G$-variety $\theta(X)$ in a similar way.) A $G \times G$-variety $X$ is self-adjoint if it admits an involutive automorphism $\Theta_{X}$ such that $\Theta_{X}(\gamma x)=\Theta(\gamma) \Theta_{X}(x)$ for all $\gamma \in G \times G$ and $x \in X$. As an easy example, any $T$-variety $X$ is a self-adjoint $T \times T$-variety for the action $\left(t_{1}, t_{2}\right) \cdot x=t_{1} t_{2}^{-1} x$, and the identity automorphism.

For any $G \times G$-variety $X$, the fixed point subset $X^{\operatorname{diag} T}$ is equipped with an action of $N_{G \times G}(\operatorname{diag} T) / \operatorname{diag} T=(\operatorname{diag} N)(T \times T) / \operatorname{diag} T$. Via the second 
projection, this group is isomorphic to $W T$, the semi-direct product of $T$ with $W \subset$ Aut $T$.

Finally, recall that a variety $Y$ is seminormal if every finite bijective morphism from a variety to $Y$ is an isomorphism. Any variety $X$ admits a unique (hence, equivariant) seminormalization $\pi: Y \rightarrow X$.

Definition 1.3. An affine stable reductive variety (resp. an affine reductive variety) for $G$ is a connected (resp. irreducible) affine $G \times G$-variety $X$ satisfying the following conditions:

(1) (on singularities) $X$ is seminormal,

(2) (on stabilizers) for any $x \in X$, the stabilizer $\operatorname{Stab}_{G \times G}(x)$ is connected,

(3) (on orbits) $X$ contains only finitely many $G \times G$-orbits,

(4) (group-like condition) $\left(U^{-} \times U\right) X^{\operatorname{diag} T}$ contains a dense subset of every $G \times G$-orbit.

Here we are following a well-established (but somewhat confusing) tradition started by Deligne and Mumford [DM69] with their definition of stable curves. Namely, an affine reductive variety is also a stable reductive variety but not necessarily vice versa. In other words, "stable" serves to widen the class, not to narrow it down. And since most varieties considered in this paper are affine, we will often drop the adjective "affine", and deal with (stable) reductive varieties.

The easiest reductive variety is the group $G$ itself, where $G \times G$ acts by $\left(g_{1}, g_{2}\right) \cdot g=g_{1} g g_{2}^{-1}$ : then $G \simeq(G \times G) / \operatorname{diag} G$. Moreover, $G^{\operatorname{diag} T}=T$, and the subset $\left(U^{-} \times U\right) G^{\operatorname{diag} T}=U^{-} T U=B^{-} B$ is open in $G$. Note that $G$ is self-adjoint for $\Theta_{G}: g \mapsto \theta\left(g^{-1}\right)$; moreover, $\Theta_{G}$ fixes $T$ pointwise.

If $G=T$ is a torus, then condition (4) just means that $\operatorname{diag} T$ fixes pointwise $X$. Therefore, the stable reductive varieties for $T$ are exactly the seminormal affine varieties where $T$ acts with finitely many orbits and connected isotropy groups. These are the affine stable toric varieties in the sense of Ale02.

Our first main result (Theorems 4.8 and 5.4) classifies all stable reductive varieties for $G$, in terms of stable toric varieties for $T$.

Theorem. Let $X$ be a stable reductive variety for $G$. Then $X^{\operatorname{diag} T}$ is a stable toric variety for $T$, with a compatible action of $W$ (that is, an action of $W T)$. Moreover, $X$ is self-adjoint for a unique automorphism $\Theta_{X}$ fixing pointwise $X^{\operatorname{diag} T}$.

Any $G \times G$-orbit $\mathcal{O}$ in $X$ meets $X^{\operatorname{diag} T}$ into a unique $W T$-orbit; moreover, $\operatorname{Aut}^{G \times G}(X) \simeq \operatorname{Aut}^{W T}\left(X^{\operatorname{diag} T}\right)$.

The assignment $X \mapsto X^{\operatorname{diag} T}$ defines a bijective correspondence from the stable reductive varieties (for $G$ ) to the stable toric varieties (for $T$ ) with a compatible $W$-action. Moreover, $X$ is irreducible if and only if $X^{\operatorname{diag} T} / W$ is. 
Definition 1.4. A stable reductive semigroup (resp. a reductive semigroup) for $G$ is a connected (resp. irreducible) affine $G \times G$-variety $X$ satisfying conditions (1), (2) of Definition 1.3. and equipped with a morphism $m$ : $X \times X \rightarrow X$ satisfying the following conditions:

(1) (on semigroups) $m$ is an associative multiplication law on $X$.

(2) (on equivariance) $m\left(\left(g_{1}, g\right) x_{1},\left(g, g_{2}\right) x_{2}\right)=\left(g_{1}, g_{2}\right) m\left(x_{1}, x_{2}\right)$ for all $g_{1}, g, g_{2} \in G$ and $x_{1}, x_{2} \in X$.

(3) (on invariance) $m$ is a categorical quotient for the $G$-action given by $g \cdot\left(x_{1}, x_{2}\right)=\left((1, g) x_{1},(g, 1) x_{2}\right)$.

If, in addition, $X$ is self-adjoint for an automorphism $\Theta_{X}$ such that

$$
\Theta_{X}\left(m\left(x_{1}, x_{2}\right)\right)=m\left(\Theta_{X}\left(x_{2}\right), \Theta_{X}\left(x_{1}\right)\right)
$$

for all $x_{1}, x_{2} \in X$, then $X$ is a self-adjoint reductive semigroup.

The multiplication of a stable reductive semigroup $X$ will be denoted by $\left(x_{1}, x_{2}\right) \mapsto x_{1} x_{2}$, and the $G \times G$-action by $\left(g_{1}, g_{2}, x\right) \mapsto g_{1} x g_{2}^{-1}$; this notation makes sense by the equivariance condition.

By Lemma6.1 examples of self-adjoint reductive semigroups include normal reductive monoids, that is, irreducible algebraic monoids with unit group $G$, where $G \times G$ acts by left and right multiplication. These are exactly the normal affine embeddings of the homogeneous space $(G \times G) / \operatorname{diag} G$ (see Rit98 Theorem 1 and Proposition 1).

The self-adjoint reductive semigroups turn out to be closely related to reductive varieties, as shown by our second main result (Lemma 6.2 and Theorem 6.3).

Theorem. Any (stable) self-adjoint reductive semigroup is a (stable) reductive variety.

Conversely, any reductive variety admits a structure of self-adjoint reductive semigroup, and any two such structures are conjugate by a unique automorphism.

We also characterize those stable reductive varieties that admit a structure of self-adjoint stable reductive semigroup (Proposition 6.6). The class of (not necessarily self-adjoint) stable reductive semigroups is much wider: consider, for example, the $G \times G$-variety $X=Y \times Z$, where $Y$ and $Z$ are affine $G$ varieties. Then the map $m: X \times X \rightarrow X,\left(y_{1}, z_{1}, y_{2}, z_{2}\right) \mapsto\left(y_{1}, z_{2}\right)$ satisfies conditions (1) and (2) of Definition [1.4. If, in addition, the categorical quotient of $X$ by the action of $\operatorname{diag} G$ is a point, then $m$ satisfies (3) as well.

Notation 1.5. (on schemes). By a scheme, we mean a separated Noetherian scheme over $k$; morphisms (resp. products) of schemes are understood to be $k$-morphisms (resp. products over $k$ ). For any point $s$ of a scheme $S$, we denote by $k(s)$ the residue field at $s$. The choice of an algebraic closure $k(\bar{s}) \supseteq k(s)$ defines a geometric point $\bar{s}$ over $s$.

Definition 1.6. A family of affine (stable) reductive varieties over a scheme $S$ is a scheme $\mathcal{X}$ equipped with a morphism $\pi: \mathcal{X} \rightarrow S$ and with an action 
of the constant group scheme $G \times G \times S$ over $S$, satisfying the following conditions:

(1) $\pi$ is flat and affine.

(2) The geometric fiber $\mathcal{X}_{\bar{s}}$ at every geometric point $\bar{s}$ is a (stable) reductive variety for $(G \times G)(k(\bar{s}))$.

In that case, $\pi$ is the categorical quotient by $G \times G$ (Lemma 7.2). There are obvious notions of trivial (resp. locally trivial) families, and one sees easily that any family of toric varieties is locally trivial (Lemma 7.4). Clearly, this does not extend to families of reductive varieties. But any such family $\pi: \mathcal{X} \rightarrow S$, where $S$ is integral, becomes locally trivial after some étale base change (Corollary [7.9); this defines the general fiber of $\pi$.

Our third main result concerns moduli. For a fixed $G$-module $V$, we construct a fine moduli space $\mathcal{M}_{h, V}$ of $G$-subvarieties of $V$ that have a fixed Hilbert function $h$, and we prove that $\mathcal{M}_{h, V}$ is quasiprojective (Theorem (7.6). We also describe all one-parameter flat degenerations of stable reductive varieties (Proposition 7.15).

Our fourth main result (Theorem 7.18) provides a local model for families of reductive varieties over an integral scheme, with a prescribed general fiber. Its starting point is the construction of a remarkable class of such families, by Vinberg Vin95. To each semisimple group $G_{0}$, he associated its "enveloping monoid" $\operatorname{Env}\left(G_{0}\right)$. This is a normal reductive monoid having $G_{0}$ as the derived subgroup of its unit group, and such that the categorical quotient $\pi: \operatorname{Env}\left(G_{0}\right) \rightarrow \operatorname{Env}\left(G_{0}\right) / /\left(G_{0} \times G_{0}\right)$ is flat, with reduced and irreducible geometric fibers. Moreover, any reductive monoid satisfying these properties is obtained from $\operatorname{Env}\left(G_{0}\right)$ by base change. In addition, $\operatorname{Env}\left(G_{0}\right) / /\left(G_{0} \times G_{0}\right)$ is the affine space $\mathbb{A}^{r}$, where $r$ is the rank of $G_{0}$; and the fiber of $\pi$ at the identity is $G_{0}$. Hence $\pi$ is a family of reductive varieties for $G_{0}$, with general fiber $G_{0}$ itself.

Given any reductive variety $X$ for $G$, a variant of Vinberg's construction yields a family of reductive varieties $\pi_{X}: \mathcal{V}_{X} \rightarrow \mathbb{A}^{r}$ with general fiber $X$, the Vinberg family of $X$; here $r$ is the semisimple rank of $G$ (see 7.5 for details).

Theorem. Any family of reductive varieties over an integral scheme is locally a base change of the Vinberg family of its general fiber.

\section{General criteria}

2.1. Seminormality and connectedness of isotropy groups. We will obtain criteria for conditions (1), (2) of Definition 1.3 to hold; we begin with some additional notation on groups and varieties.

The weight lattice of $G$ is the character group of $T$, denoted by $\Lambda$; let $\Lambda_{\mathbb{R}}$ be the corresponding real vector space. The positive Weyl chamber of $\Lambda_{\mathbb{R}}$ associated with $B$ is denoted by $\Lambda_{\mathbb{R}}^{+}$; then $\Lambda^{+}=\Lambda \cap \Lambda_{\mathbb{R}}^{+}$is the set of dominant weights. For each $\lambda \in \Lambda^{+}$, let $V_{\lambda}$ be the simple $G$-module with highest weight $\lambda$. 
For any $G$-variety $X$, the algebra of regular functions $k[X]$ is a rational $G$-module; thus, it decomposes as a direct sum of simple submodules $V_{\lambda}$, with (possibly infinite) positive multiplicities. The set of all such $\lambda$ is the weight set $\Lambda_{X}^{+}$; let $\Lambda_{X}$ (resp. $C_{X}$ ) be the subgroup of $\Lambda$ (resp. the cone of $\left.\Lambda_{\mathbb{R}}\right)$ generated by $\Lambda_{X}^{+}$. Then $\Lambda_{X}^{+} \subseteq \Lambda^{+} \cap C_{X}$; this inclusion may be strict.

If, in addition, $X$ is affine, then the invariant subalgebra $k[X]^{G}$ is finitely generated. We denote by $X / / G$ the corresponding affine variety; the corresponding morphism $p: X \rightarrow X / / G$ is the categorical quotient by $G$. The invariant subalgebra $k[X]^{U}$ is finitely generated as well; moreover, $T$ acts on $k[X]^{U}$ and hence on the associated variety $X / / U$. For any $\lambda \in \Lambda$, let $k[X]_{\lambda}^{U}$ be the $\lambda$-weight space in the $T$-module $k[X]^{U}$. Then $k[X]_{\lambda}^{U} \simeq \operatorname{Hom}^{G}\left(V_{\lambda}, k[X]\right)$; in particular, $k[X]_{0}^{U}=k[X]^{G}$. Any $k[X]_{\lambda}^{U}$ is a finitely generated $k[X]^{G}$-module; its dimension (as a $k$-vector space) is the multiplicity of the $G$-module $V_{\lambda}$ in $k[X]$ if $\lambda \in \Lambda^{+}$, and is 0 otherwise.

We say that the affine $G$-variety $X$ is multiplicity-free (resp. multiplicityfinite, multiplicity-bounded) if all these multiplicities are 0 or 1 (resp. finite, bounded), see Gro97 $\$ 11$. Note that $X$ is multiplicity-finite if and only if $k[X]^{G}$ is finite-dimensional as a $k$-vector space.

Recall that a $G$-variety $X$ is spherical if $X$ is normal and contains a dense $B$-orbit; then it contains only finitely many $B$-orbits, see Gro97. Theorem 22.6. The affine spherical varieties are exactly the irreducible normal, multiplicity-free varieties, by Gro97] Theorem 11.1.

Lemma 2.1. Let $X$ be an affine $G$-variety.

(1) $X$ is connected if and only if $X / / G$ is. As a consequence, any multiplicity-free variety is connected.

(2) If $X$ is irreducible (resp. normal, seminormal), then so is $X / / G$.

(3) $X$ is irreducible (resp. normal, seminormal) if and only if $X / / U$ is.

Proof. The assertions on connectedness, irreducibility, and normality are well-known, see e.g. Gro97 §18.

(2) Assume that $X$ is seminormal. Let $\pi: Y \rightarrow X / / G$ be the seminormalization. Let $p: X \rightarrow X / / G$ be the quotient morphism, and form the cartesian square

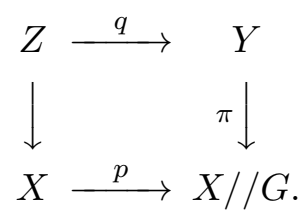

Let $Z_{\text {red }}$ be the reduced subscheme of $Z$. Then the induced map $Z_{\text {red }} \rightarrow X$ is finite and bijective, hence an isomorphism. Moreover, $G$ acts on $Z$, and $q: Z \rightarrow Y$ is the quotient map (since $G$ is reductive). Thus, this map factors through an isomorphism $Z_{\text {red }} / / G \rightarrow Y$ (since $Y$ is reduced). It follows that $\pi: Y \rightarrow X / / G$ is an isomorphism as well. 
(3) If $X$ is seminormal, then so is $X \times G / / U$ (since $G / / U$ is normal.) Thus, $(X \times G / / U) / / G$ is seminormal by $(1)$. But $(X \times G / / U) / / G \simeq X / / U$ by Gro97] Theorem 9.1, so that $X / / U$ is seminormal.

Conversely, assume that $X / / U$ is seminormal and consider the seminormalization $\pi: Y \rightarrow X$. Then $G$ acts on $Y$ and the map $\pi \times$ id $: Y \times G / / U \rightarrow$ $X \times G / / U$ is the seminormalization. It follows that the induced map $(Y \times G / / U) / / G \rightarrow(X \times G / / U) / / G$ is finite and bijective. By Gro97 Theorem 9.1 again, this means that the map $\pi / / U: Y / / U \rightarrow X / / U$ is finite and bijective. Since $X / / U$ is seminormal, $\pi / / U$ is an isomorphism; since $Y$ is a $G$-variety, it follows that $\pi$ is an isomorphism.

Lemma 2.2. Let $X$ be an affine spherical $G$-variety. Then:

(1) $C_{X}$ is a rational polyhedral convex cone, and $\Lambda_{X}^{+}=C_{X} \cap \Lambda_{X}$.

(2) Any closed irreducible $G$-subvariety $Y$ of $X$ is uniquely determined by $\Lambda_{Y}^{+}$. Moreover, $Y$ is normal, and there exists a unique face $F$ of $C_{X}$ such that $\Lambda_{Y}^{+}=\Lambda_{X}^{+} \cap F$.

(3) If $\Lambda_{X}^{+}$is saturated in $\Lambda$, then each isotropy group of $G$ in $X$ is connected.

Proof. Note that $X / / U$ is an affine toric variety for the torus with character group $\Lambda_{X}$ (a quotient of $T$ ). By the theory of toric varieties (see e.g. [Ful93]), this implies (1), and (by considering $Y / / U$ ) the second and third assertions of (2). Since $X$ is multiplicity-free and the ideal of $Y$ in $k[X]$ is $G$-invariant, this ideal is uniquely determined by $\Lambda_{Y}^{+}$. This completes the proof of (2).

(3) By (2), $\Lambda_{Y}^{+}$is saturated in $\Lambda$ for any closed irreducible $G$-subvariety $Y$. Thus, it suffices to show that the isotropy group $H$ of a point of the open $G$-orbit is connected. Let $H^{0}$ be the connected component of the identity in $H$. Then we have a dominant $G$-equivariant map $G / H^{0} \rightarrow X$. Let $\tilde{X}$ be the normalization of $X$ in the field of rational functions on $G / H^{0}$. This is an affine spherical $G$-variety, and the algebra $k[\tilde{X}]^{U}$ is integral over $k[X]^{U}$. It follows that any $\chi \in \Lambda_{\tilde{X}}^{+}$admits a positive integral multiple in $\Lambda_{X}^{+}$. Since $\Lambda_{X}^{+}$ is saturated in $\Lambda$, this implies $\Lambda_{\tilde{X}}^{+}=\Lambda_{X}^{+}$, whence $\tilde{X}=X$, and $H^{0}=H$.

Lemma 2.3. Let $X$ be an affine $T$-variety. Then $X$ is multiplicity-bounded if and only if every irreducible component is multiplicity-free; in that case, $X$ contains only finitely many $T$-orbits.

If, in addition, $X$ is multiplicity-free, then the following conditions are equivalent:

(1) $X$ is seminormal, with connected isotropy groups.

(2) $\Lambda_{X}^{+}$is saturated in $\Lambda$.

Then any $T$-orbit closure in $X$ is normal.

Proof. If every irreducible component of $X$ is multiplicity-free, then $X$ is clearly multiplicity-bounded. Conversely, let $X$ be multiplicity-bounded and let $Y$ be an irreducible component. If we can find linearly independent $T$-eigenvectors $\varphi, \psi \in k[Y]$ of the same weight $\lambda$, then for any positive 
integer $n$, the monomials $\varphi^{n}, \varphi^{n-1} \psi, \ldots, \varphi \psi^{n-1}, \psi^{n}$ are linearly independent $T$-eigenvectors of weight $n \lambda$. This contradicts the assumption that $X$ is multiplicity-bounded; thus, $Y$ is multiplicity-free. Using Lemma 2.2 it follows that $X$ contains only finitely many orbits.

$(1) \Rightarrow(2)$ : Let $\left(X_{i}\right)$ be the (finite) family of all closed irreducible $T$ subvarieties of $X$, and let $\left(\tilde{X}_{i}\right)$ be the family of their normalizations. These form a direct system, with direct limit $X$ by Ale02 Theorem 2.3.14. In other words, $k[X]=\lim _{i} k\left[\tilde{X}_{i}\right]$. Since each $\tilde{X}_{i}$ is a toric variety for a quotient of $T$ by a subtorus, ${\overleftarrow{\Lambda_{\tilde{X}}}}^{+}$is saturated in $\Lambda$. It follows that the same holds for $\Lambda_{X}^{+}$.

$(2) \Rightarrow(1)$ : Let $Y$ be a closed irreducible $T$-subvariety of $X$. Then the assumptions imply that $Y$ is normal and multiplicity-free, and that $\Lambda_{Y}^{+}$is saturated in $\Lambda$. Thus, $Y$ is a toric variety for the quotient of $T$ by a subtorus. So all isotropy subgroups of $X$ are connected.

Let $Y$ be the direct limit of all closed irreducible $T$-subvarieties of $X$. Then $Y$ is seminormal by Ale02 Corollary 2.3.10. The natural morphism $\pi: Y \rightarrow X$ is clearly finite and bijective; since $X$ is multiplicity-free and $\Lambda_{X}^{+}$is saturated, $\pi$ is an isomorphism. In other words, $X$ is seminormal. Moreover, every $T$-orbit closure is normal.

Combining Lemmas 2.1, 2.2 and 2.3, we obtain the following criterion for an affine $G$-variety to satisfy conditions (1), (2) and (3) of Definition 1.3

Proposition 2.4. Any multiplicity-bounded $G$-variety $X$ contains only finitely many $G$-orbits, and these are spherical. As a consequence, $X$ contains only finitely many $B$-orbits.

If, in addition, $X$ is multiplicity-free and $\Lambda_{X}^{+}$is saturated in $\Lambda$, then $X$ is connected and seminormal, the $G$-orbit closures are normal, and their isotropy groups are connected.

We will also need the following observation.

Lemma 2.5. Let $X$ be an irreducible, multiplicity-free $G$-variety and let $\nu: \tilde{X} \rightarrow X$ be the normalization map. If all isotropy groups are connected, then $\nu$ is bijective.

Proof. Consider an orbit $\mathcal{O}$ in $X$, and an orbit $\tilde{\mathcal{O}}$ in $\nu^{-1}(\mathcal{O})$. Then the restriction $\nu: \tilde{\mathcal{O}} \rightarrow \mathcal{O}$ is an isomorphism, since the isotropy groups of $\mathcal{O}$ are connected. Hence $\nu^{-1}(\mathcal{O})$ is a union of finitely many copies of $\mathcal{O}$.

On the other hand, for any orbit $\tilde{\mathcal{O}}$ in $\tilde{X}$, with closure $Y$, we have $\Lambda_{\tilde{\mathcal{O}}}=$ $\Lambda_{Y}$. By Lemma 2.2, it follows that the linear span of $\Lambda_{\tilde{\mathcal{O}}}$ intersects $C_{X}$ along a face, which uniquely determines $Y$ and hence $\tilde{\mathcal{O}}$. Thus, any two distinct orbits in $\tilde{X}$ are non-isomorphic as $G$-varieties. It follows that $\nu$ is bijective.

2.2. Finiteness of number of orbits and group-like condition. We will obtain a representation-theoretic criterion for a variety to satisfy conditions (3) and (4) of Definition [1.3. For this, we introduce some notation on representations. 
The simple $G \times G$-modules are the $V_{\lambda} \otimes_{k} V_{\mu}$ where $\lambda, \mu \in \Lambda^{+}$; those containing non-zero fixed points of $\operatorname{diag} G$ are the End $V_{\lambda}=V_{\lambda}^{*} \otimes_{k} V_{\lambda}$. Note that $\theta\left(V_{\lambda}\right) \simeq V_{\lambda}^{*}$, so that $\Theta\left(V_{\lambda} \otimes_{k} V_{\mu}\right) \simeq V_{\mu}^{*} \otimes_{k} V_{\lambda}^{*}$; in particular, $\Theta\left(\right.$ End $\left.V_{\lambda}\right) \simeq$ End $V_{\lambda}$ as $G \times G$-modules.

Definition 2.6. An affine $G \times G$-variety $X$ is diagonal if every simple submodule of the $G \times G$-module $k[X]$ is isomorphic to some End $V_{\lambda}$. Equivalently, the weights of the $T \times T$-module $k[X]^{U^{-} \times U}$ are of the form $(-\lambda, \lambda)$, where $\lambda \in \Lambda^{+}$. We then identify the weight set of $X$ to a subset of $\Lambda^{+}$, via the second projection; this identifies $\Lambda_{X}$ to a subgroup of $\Lambda$.

Proposition 2.7. For an affine $G \times G$-variety $X$, the following conditions are equivalent:

(1) $X$ contains only finitely many $G \times G$-orbits, and $\left(U^{-} \times U\right) X^{\operatorname{diag} T}$ contains a dense subset of any such orbit.

(2) $X$ is diagonal and multiplicity-bounded.

(3) $X$ contains only finitely many $B^{-} \times B$-orbits, and any such orbit which is open in its $G \times G$-orbit contains fixed points of $\operatorname{diag} T$.

Proof. (1) $\Rightarrow(2)$ Every $T \times T$-eigenvector $f \in k[X]^{U^{-} \times U}$ is uniquely determined by its restriction to $X^{\operatorname{diag} T}$. Thus, the weight of $f$ is $(-\lambda, \lambda)$ for some $\lambda \in \Lambda^{+}$. Moreover, for any $G \times G$-orbit $\mathcal{O}$, the subset $\mathcal{O}^{\operatorname{diag} T}$ contains only finitely many $T \times T$-orbits (since $T \times T$ is the centralizer of $\operatorname{diag} T$ in $G \times G$ ). As a consequence, $X^{\operatorname{diag} T}$ contains only finitely many $T \times T$-orbits. Hence the multiplicities of all $T \times T$-weight spaces in $k\left[X^{\operatorname{diag} T}\right]$ are bounded, and the same holds for the multiplicities of all weight spaces in $k[X]^{U^{-} \times U}$.

$(2) \Rightarrow(3)$ Let $Y$ be a closed irreducible $G \times G$-subvariety of $X$. Then $Y$ is multiplicity-free, by Lemma 2.3. It follows that $Y$ contains only finitely many $B^{-} \times B$-orbits, and hence an open $B^{-} \times B$-orbit $Y_{0}$. Moreover, the weights of the $T \times T$-eigenvectors in $k[Y]^{U^{-} \times U}$, and hence in $k\left[Y_{0}\right]^{U^{-} \times U}$, are of the form $(-\lambda, \lambda)$. Thus, $\operatorname{diag} T$ fixes pointwise the quotient $Y_{0} /\left(U^{-} \times U\right)$. This implies easily that $Y_{0}$ contains a $\operatorname{diag} T$-fixed point.

$(3) \Rightarrow(1)$ is obvious.

The local structure of a variety satisfying these conditions is described by the following

Lemma 2.8. Let $X$ be a diagonal, multiplicity-bounded $G \times G$-variety.

(1) Let $f \in k[X]^{U^{-} \times U}$ be a $T \times T$-eigenvector of weight $\lambda$. Then the stabilizer of the line $k f$ is a product $P^{-} \times P$, where $P \supseteq B$ and $P^{-} \supseteq B^{-}$are opposite parabolic subgroups. Let $L=P \cap P^{-} \supseteq T$ be their common Levi subgroup. Then there exists a locally closed affine $L \times L$-subvariety $Z \subseteq X$ such that the map

$$
R_{u}\left(P^{-}\right) \times R_{u}(P) \times Z \rightarrow X,\left(g_{1}, g_{2}, x\right) \mapsto\left(g_{1}, g_{2}\right) x
$$

is an open immersion with image the principal open subset $X_{f}$. Moreover, $Z$ is a diagonal, multiplicity-bounded $L \times L$-variety. 
(2) If, in addition, $X$ is multiplicity-free, then so is $Z$.

(3) For any open $B^{-} \times B$-orbit $X_{0}$ in $X$, there exists $f$ as in (1) such that $X_{f}=X_{0}$. Then $P$ and $L$ depend only on $X_{0}$, and $Z=X_{0}^{\operatorname{diag} T}$ is a unique $T \times T$-orbit, fixed pointwise by $[L, L] \times[L, L]$, where $[L, L]$ is the derived subgroup of $L$.

Proof. (1) The first assertion follows from Kno94] Theorem 2.3. Together with Proposition 2.7, it implies the second assertion.

(2) Let $u, v$ be $T \times T$-eigenvectors in $k[Z]^{\left(U^{-} \cap L\right) \times(U \cap L)}$, of the same weight $\mu$. We may regard them as $T \times T$-eigenvectors in $k\left[X_{f}\right]^{U^{-} \times U}$. Thus, there exists a positive integer $n$ such that $u f^{n}, v f^{n}$ lift to $T \times T$-eigenvectors $\tilde{u}, \tilde{v}$ in $k[X]^{U^{-} \times U}$, under the localization $k[X] \rightarrow k\left[X_{f}\right]$. Then $\tilde{u}, \tilde{v}$ have weight $\mu+n \lambda$. Since $X$ is multiplicity-free, $\tilde{v} \in k^{*} \tilde{u}$, so that $v \in k^{*} u$.

(3) We may choose $f$ as in (1) such that $f$ vanishes everywhere on $X-X_{0}$; then $X_{f}=X_{0}$. By Kno94 Proposition 2.4, the corresponding $Z$ is fixed pointwise by $[L, L] \times[L, L]$. Since $X_{0}$ is a unique $B^{-} \times B$-orbit, isomorphic to $R_{u}\left(P^{-}\right) \times R_{u}(P) \times Z$, it follows that $Z$ is a unique $T \times T$-orbit, containing $X_{0}^{\operatorname{diag} T}$. The latter is $T \times T$-invariant and non-empty; thus, $Z=X_{0}^{\operatorname{diag} T}$.

\section{Orbits in Stable Reductive VARIETIES}

3.1. Isotropy groups. We will determine those quasi-affine homogeneous $G \times G$-varieties $\mathcal{O}$ that satisfy the "group-like" condition (4) of Definition 1.3. By Proposition 2.7, we may find $x \in \mathcal{O}^{\operatorname{diag} T}$ such that $\left(B^{-} \times B\right) x$ is open in $\mathcal{O}$. Then the isotropy group $H=\operatorname{Stab}_{G \times G}(x)$ contains $\operatorname{diag} T$, and the product $\left(B^{-} \times B\right) H$ is open in $G \times G$. To describe $H$, we need additional notation on subgroups of $G$.

Let $\Phi \subset \Lambda$ be the root system of $(G, T)$, with its subset of positive roots $\Phi^{+}$(the roots of $(B, T)$ ), and corresponding subset of simple roots $\Pi$. The coroot of each $\alpha \in \Phi$ is denoted by $\check{\alpha}$. For any subset $I \subseteq \Pi$, let $P_{I} \supseteq B$ be the corresponding parabolic subgroup of $G$, and let $P_{I}^{-} \supseteq B^{-}$be the opposite parabolic subgroup; then $L_{I}=P_{I} \cap P_{I}^{-}$is a Levi subgroup of both, containing $T$. Let $W_{I} \subseteq W$ (resp. $\left.\Phi_{I} \subseteq \Phi\right)$ be the Weyl group (resp. the root system) of $\left(L_{I}, T\right)$ and let $Z\left(L_{I}\right)=Z_{I}$ be the center of $L_{I}$. Then $Z_{I} \subseteq T$ is the intersection of the kernels of the characters $\alpha \in I$.

Proposition 3.1. Let $H \subseteq G \times G$ be a closed subgroup satisfying the following conditions.

(1) $H$ contains $\operatorname{diag} T$.

(2) $\left(B^{-} \times B\right) H$ is open in $G \times G$.

(3) The homogeneous space $G \times G / H$ is quasi-affine.

Then there exist a subset $I \subseteq \Pi$, union of two orthogonal subsets $J$ and $K$, and a subgroup $H_{J} \subseteq G$ such that:

(1) $\left[L_{J}, L_{J}\right] \subseteq H_{J} \subseteq\left[L_{J}, L_{J}\right] Z_{K}$, and

(2) $H$ is conjugate in $T \times T$ to $\left.R_{u}\left(P_{I}\right) H_{J} \times R_{u}\left(P_{I}^{-}\right) H_{J}\right) \operatorname{diag} L_{K}$. 
Moreover, $N_{G \times G}(H)=H\left(Z_{K} \times Z_{K}\right)$.

Proof. Let $P \supseteq B$ be as in Lemma 2.8 (3). By that Lemma, the multiplication map $R_{u}\left(P^{-}\right) \times R_{u}(P) \times(L \times L) H \rightarrow G \times G$ is an open immersion; moreover, $(L \times L) \cap H$ contains $([L, L] \times[L, L]) \operatorname{diag} T$. Write $P=P_{J}$ for some $J \subseteq \Pi$; then $\left(L_{J} \times L_{J}\right) \cap H=\left(H_{J} \times H_{J}\right) \operatorname{diag} T$ for a unique closed subgroup $H_{J}$ of $L_{J}$, containing $\left[L_{J}, L_{J}\right]$. Therefore, one obtains a decomposition of the Lie algebra of $G \times G$ :

$$
\operatorname{Lie}(G \times G)=\operatorname{Lie}\left(R_{u}\left(P_{J}^{-}\right) \times R_{u}\left(P_{J}\right)\right) \bigoplus\left(\operatorname{Lie}\left(L_{J} \times L_{J}\right)+\operatorname{Lie}(H)\right),
$$

and $\operatorname{Lie}\left(L_{J} \times L_{J}\right) \cap \operatorname{Lie}(H)=\operatorname{Lie}\left(H_{J} \times H_{J}\right)+\operatorname{diag} \operatorname{Lie}(T)$. On the other hand, one has the decomposition

$$
\operatorname{Lie}(G \times G)=\operatorname{Lie}\left(R_{u}\left(P_{J}^{-}\right) \times R_{u}\left(P_{J}\right)\right) \oplus \operatorname{Lie}\left(L_{J} \times L_{J}\right) \oplus \operatorname{Lie}\left(R_{u}\left(P_{J}\right) \times R_{u}\left(P_{J}^{-}\right)\right) .
$$

For any $\alpha \in \Phi$, choose a root vector $e_{\alpha} \in \operatorname{Lie}(G)_{\alpha}$. Since $\operatorname{Lie}(H)$ is stable under $\operatorname{diag}(T)$, it decomposes as a sum of weight spaces; thus, $\operatorname{Lie}(H)$ has a basis consisting of elements of $\operatorname{Lie}(T \times T)$ and of certain $\left(u_{\alpha} e_{\alpha}, v_{\alpha} e_{\alpha}\right)$ where $u_{\alpha}, v_{\alpha}$ are scalars. In fact, the preceding decompositions imply that

$$
\operatorname{Lie}(H)=\operatorname{Lie}\left(\left(L_{J} \times L_{J}\right) \cap H\right) \oplus \bigoplus_{\alpha \in \Phi^{+-} \Phi_{J}} k\left(e_{\alpha}, c_{\alpha} e_{\alpha}\right) \oplus \bigoplus_{\alpha \in \Phi^{+-} \Phi_{J}} k\left(d_{\alpha} e_{-\alpha}, e_{-\alpha}\right)
$$

for certain scalars $c_{\alpha}, d_{\alpha}$.

We claim that $c_{\alpha}=d_{\alpha}$ for any $\alpha \in \Phi^{+}-\Phi_{I}$. To see this, set $\left[e_{\alpha}, e_{-\alpha}\right]=h_{\alpha}$; then $h_{\alpha} \in \operatorname{Lie}(T)$, and $\left[h_{\alpha}, e_{\alpha}\right]$ is a non-zero scalar multiple of $e_{\alpha}$. One has

$$
\left[\left(e_{\alpha}, c_{\alpha} e_{\alpha}\right),\left(d_{\alpha} e_{-\alpha}, e_{-\alpha}\right)\right]=\left(d_{\alpha} h_{\alpha}, c_{\alpha} h_{\alpha}\right) \in \operatorname{Lie}(H) .
$$

If $c_{\alpha} \neq d_{\alpha}$, then $\operatorname{Lie}(H)$ contains both $\left(h_{\alpha}, 0\right)$ and $\left(0, h_{\alpha}\right)$, since $\operatorname{Lie}(H)$ contains $\operatorname{diag} \operatorname{Lie}(T)$. Thus, $\operatorname{Lie}(H)$ contains $\left[\left(h_{\alpha}, 0\right),\left(e_{\alpha}, c_{\alpha} e_{\alpha}\right)\right]=\left(\left[h_{\alpha}, e_{\alpha}\right], 0\right)$, that is, $\left(e_{\alpha}, 0\right) \in \operatorname{Lie}(H)$. So $c_{\alpha}=0$; likewise, $d_{\alpha}=0$, a contradiction. The claim is proved.

Next we consider the images $H_{1}, H_{2}$ of $H$ under the projections $p_{1}, p_{2}$ : $G \times G \rightarrow G$. By the decomposition of $\operatorname{Lie}(H)$ into $\operatorname{diag} T$-eigenspaces and the preceding claim, $H_{1}$ is a parabolic subgroup containing $B$, and the set of roots of $\left(H_{1}, T\right)$ equals

$$
\Phi^{+} \cup \Phi_{J} \cup\left\{-\alpha \mid \alpha \in \Phi^{+}-\Phi_{J}, c_{\alpha} \neq 0\right\} .
$$

Moreover, $H_{2}$ is the opposite parabolic subgroup to $H_{1}$ that contains $T$. Hence $H_{1}=P_{I}$ and $H_{2}=P_{I}^{-}$for some subset $I \subseteq \Pi$ containing $J$; then

$$
\Phi_{I}^{+}=\Phi_{J}^{+} \cup\left\{\alpha \in \Phi^{+}-\Phi_{J} \mid c_{\alpha} \neq 0\right\} .
$$

Let $E=\left\{\alpha \in \Phi^{+}-\Phi_{J} \mid c_{\alpha} \neq 0\right\}$. If $\alpha \in E$ and $\beta \in \Phi_{J}^{+}$, then $\alpha+\beta \notin$ $\Phi_{I}^{+}$(otherwise, since $\left[\left(e_{\alpha}, c_{\alpha} e_{\alpha}\right),\left(e_{\beta}, 0\right)\right] \in \operatorname{Lie}(H)$, we obtain $\left(e_{\alpha+\beta}, 0\right) \in$ $\operatorname{Lie}(H)$; multiplying by $\left(e_{-\beta}, 0\right) \in \operatorname{Lie}(H)$, it follows that $\left(e_{\alpha}, 0\right) \in \operatorname{Lie}(H)$, a contradiction). Since $\Phi_{I}^{+}$is the disjoint union of $\Phi_{J}^{+}$and $E$, there exists a subset $K \subseteq I$ orthogonal to $J$, such that $E=\Phi_{K}^{+}$. Moreover, $I=J \cup K$. 
Together with the decomposition of $\operatorname{Lie}(H)$ into $\operatorname{diag} T$-eigenspaces, this implies that the connected component $H^{0}$ satisfies

$$
H^{0}=\left(R_{u}\left(P_{I}\right) H_{J}^{0} \times R_{u}\left(P_{I}^{-}\right) H_{J}^{0}\right) H_{K},
$$

where $H_{K}$ is the closed connected subgroup of $G \times G$ with Lie algebra spanned by $\operatorname{Lie}(\operatorname{diag} T)$ and by the $\left(e_{\alpha}, c_{\alpha} e_{\alpha}\right),\left(c_{\alpha} e_{-\alpha}, e_{-\alpha}\right)\left(\alpha \in \Phi_{K}^{+}\right)$. Then $H_{K} \subseteq L_{K} \times L_{K}$, and both projections $H_{K} \rightarrow L_{K}$ are isomorphisms: $H_{K}$ is the graph of an automorphism of the group $L_{K}$ fixing $T$ pointwise. Such an automorphism is the conjugation by an element of $T$; therefore, we may assume that $H^{0}=\left(R_{u}\left(P_{I}\right) H_{J}^{0} \times R_{u}\left(P_{I}^{-}\right) H_{J}^{0}\right) \operatorname{diag} L_{K}$. Moreover, $H_{J}^{0}=$ $\left[L_{J}, L_{J}\right]\left(T \cap H_{J}^{0}\right)$, and by considering the adjoint action of $T \cap H_{J}^{0}$ on $\left(e_{\alpha}, e_{\alpha}\right)$ where $\alpha \in K$, one obtains that $\alpha$ vanishes on $T \cap H_{J}^{0}$. Thus, $\left[L_{J}, L_{J}\right] \subseteq$ $H_{J}^{0} \subseteq\left[L_{J}, L_{J}\right] Z_{K}$.

Let $\tilde{H}$ be the normalizer of $H^{0}$ in $G \times G$. Then $\tilde{H}$ normalizes the unipotent radical $R_{u}\left(P_{I}\right) \times R_{u}\left(P_{I}^{-}\right)$of $H^{0}$, so that $\tilde{H} \subseteq P_{I} \times P_{I}^{-}$. In fact, $\tilde{H}=$ $\left(R_{u}\left(P_{I}\right) \times R_{u}\left(P_{I}^{-}\right)\right)\left(\tilde{H} \cap\left(L_{I} \times L_{I}\right)\right)$, and $\tilde{H} \cap\left(L_{I} \times L_{I}\right)$ is the normalizer in $L_{I} \times L_{I}$ of $\left(H_{J}^{0} \times H_{J}^{0}\right) \operatorname{diag} L_{K}$. Now the preceding arguments show that $\tilde{H}=\left(R_{u}\left(P_{I}\right)\left[L_{I}, L_{I}\right] Z_{K} \times R_{u}\left(P_{I}^{-}\right)\left[L_{I}, L_{I}\right] Z_{K}\right) \operatorname{diag} L_{K}$. Since $H^{0} \subseteq H \subseteq$ $N_{G \times G}(H) \subseteq N_{G \times G}\left(H^{0}\right)=\tilde{H}$, this implies our assertions on the structure of $H$ and its normalizer.

For $H=\left(R_{u}\left(P_{I}\right) H_{J} \times R_{u}\left(P_{I}^{-}\right) H_{J}\right) \operatorname{diag} L_{K}$ as in Proposition 3.1, let

$$
T_{H}=\{t \in T \mid(t, 1) \in H\}=T \cap H_{J}
$$

and denote by $\Lambda_{H}$ the set of those characters of $T$ that vanish on $T_{H}$. Then $K \subset \Lambda_{H} \subseteq J^{\perp}$, since the coroots of elements of $J$ are one-parameter subgroups of $T_{H}$. We call the triple $\left(K, \Lambda_{H}, J\right)$ the combinatorial invariant of $H$. It characterizes the orbit $G \times G / H$, by Proposition 3.1] a criterion for that orbit to be quasi-affine will be given in Proposition 3.4 below.

Conversely, given two orthogonal subsets $J, K \subseteq \Pi$ and a subgroup $\Lambda^{\prime} \subseteq \Lambda$ such that $K \subset \Lambda^{\prime} \subseteq J^{\perp}$, let $T^{\prime}$ be the subgroup of $T$, intersection of the kernels of all the $\chi \in \Lambda^{\prime}$. Also, let $H_{J}=\left[L_{J}, L_{J}\right] T^{\prime}$ and $I=J \cup K$. Then

$$
H_{K, \Lambda^{\prime}, J}=\left(R_{u}\left(P_{I}\right) H_{J} \times R_{u}\left(P_{I}^{-}\right) H_{J}\right) \operatorname{diag} L_{K}
$$

is a subgroup of $G \times G$ containing $\operatorname{diag} T$, such that $\left(B^{-} \times B\right) H_{K, \Lambda^{\prime}, J}$ is open in $G \times G$, and with combinatorial invariant $\left(K, \Lambda^{\prime}, J\right)$. We record some easy properties of the corresponding homogeneous spaces.

Lemma 3.2. For $H=H_{K, \Lambda^{\prime}, J}$, one has:

(1) $H$ is connected $\Leftrightarrow T^{\prime}$ is connected $\Leftrightarrow \Lambda^{\prime}$ is saturated in $\Lambda$.

(2) $\operatorname{Aut}^{G \times G}(G \times G / H)=N_{G \times G}(H) / H$ is isomorphic to $Z_{K} / T_{H}$, a diagonalizable group with character group $\Lambda^{\prime} / \mathbb{Z} K$.

(3) $\operatorname{dim} G \times G / H=\operatorname{rank} \Lambda^{\prime}+\left|\Phi-\Phi_{J}\right|$.

(4) $\Theta(H)=H$, so that $\Theta$ induces an involutive automorphism $\Theta_{G \times G / H}$ fixing $(T \times T) H / H$ pointwise. This makes $G \times G / H$ a self-adjoint $G \times G$-variety. 
Proof. (1) Since $H^{0}=\left(R_{u}\left(P_{I}\right) H_{J}^{0} \times R_{u}\left(P_{I}^{-}\right) H_{J}^{0}\right) \operatorname{diag} L_{K}$, and $\left[L_{J}, L_{J}\right] \subseteq$ $H_{J}^{0} \subseteq L_{J}$, one has $H / H^{0} \simeq H_{J} / H_{J}^{0} \simeq\left(T \cap H_{J}\right) /\left(T \cap H_{J}\right)^{0}=T^{\prime} / T^{\prime 0}$. Moreover, $T^{\prime}\left(\operatorname{resp} . T^{\prime 0}\right)$ is the intersection of the kernels of all characters in $\Lambda^{\prime}$ (resp. in the saturation of $\Lambda^{\prime}$ in $\Lambda$ ).

(2), (3) and (4) are straightforward.

3.2. Algebras of regular functions. We study the algebra $k[G \times G / H]$, where $H=H_{K, \Lambda^{\prime}, J}$. We begin with the simplest case where $H=\operatorname{diag} G$, that is, $J=\emptyset, \Lambda^{\prime}=\Lambda$, and $K=\Pi$. As is well-known, the algebra $k[G \times G / \operatorname{diag} G] \simeq k[\operatorname{diag} G]$ is spanned by matrix coefficients of simple $G$-modules. Specifically, the direct sum of the maps

$$
\text { End } V_{\lambda} \rightarrow k[G], u \mapsto\left(g \mapsto \operatorname{Tr}_{V_{\lambda}}(u g)\right)
$$

yields an isomorphism of $G \times G$-modules: $\bigoplus_{\lambda \in \Lambda^{+}}$End $V_{\lambda} \simeq k[G]$.

To describe the multiplication in $k[G]$, consider the decomposition of tensor products of $G$-modules:

$$
V_{\lambda} \otimes_{k} V_{\mu} \simeq \bigoplus_{\nu} N_{\lambda \mu}^{\nu} \otimes_{k} V_{\nu}
$$

where $N_{\lambda \mu}^{\nu}=\operatorname{Hom}^{G}\left(V_{\nu}, V_{\lambda} \otimes_{k} V_{\mu}\right)$. This defines $G \times G$-equivariant projections

$$
p_{\lambda \mu}^{\nu}: \text { End } V_{\lambda} \otimes_{k} \text { End } V_{\mu} \rightarrow \text { End } N_{\lambda \mu}^{\nu} \otimes_{k} \text { End } V_{\nu} .
$$

Then the multiplication in $k[G]$ of any $f_{\lambda} \in$ End $V_{\lambda}$ and $f_{\mu} \in$ End $V_{\mu}$ is given by

$$
f_{\lambda} \cdot f_{\mu}=\sum_{\nu} \operatorname{Tr}_{1}\left(p_{\lambda \mu}^{\nu}\left(f_{\lambda} \otimes f_{\mu}\right)\right),
$$

where $\operatorname{Tr}_{1}$ : End $N_{\lambda \mu}^{\nu} \otimes_{k}$ End $V_{\nu} \rightarrow$ End $V_{\nu}$ is the trace in the first summand.

For any $\lambda \in \Lambda^{+}$, let $\chi_{\lambda}: g \mapsto \operatorname{Tr}_{V_{\lambda}}(g)$ be the corresponding character. Then the $\chi_{\lambda}, \lambda \in \Lambda^{+}$, are a basis of the space $k[G]^{\operatorname{diag} G}$ of conjugationinvariant functions. The multiplication of characters is given by

$$
\chi_{\lambda} \cdot \chi_{\mu}=\sum_{\nu} c_{\lambda \mu}^{\nu} \chi_{\nu}
$$

where the $c_{\lambda \mu}^{\nu}=\operatorname{dim} N_{\lambda \mu}^{\nu}$ are the "Littlewood-Richardson coefficients". Note that $c_{\lambda \mu}^{\nu}=0$ unless $\lambda+\mu-\nu$ is a linear combination of elements of $\Pi$ with nonnegative integer coefficients; we write $\nu \leq_{\Pi} \lambda+\mu$. In particular, the product End $V_{\lambda}$. End $V_{\mu}$ is the sum of those End $V_{\nu}$ such that $c_{\lambda \mu}^{\nu} \neq 0$.

We now generalize this to any homogeneous space $G \times G / H_{K, \Lambda^{\prime}, J}$. For this, we construct analogues of matrix coefficients.

For any simple $G$-module $V_{\lambda}$, let $V_{\lambda, K}$ be the sum of the $T$-weight spaces in $V_{\lambda}$ associated with those weights $\chi$ such that $\chi \leq_{K} \lambda$, and let $V_{\lambda}^{K}$ be the sum of all other weight spaces. Then $V_{\lambda, K}=V_{\lambda}^{R_{u}\left(P_{K}\right)}$ is a simple $L_{K^{-}}$ module with highest weight $\lambda$. Moreover, $V_{\lambda}^{K}$ is stable under $P_{K}^{-}$, and $V_{\lambda}=V_{\lambda, K} \oplus V_{\lambda}^{K}$. Let

$$
p_{\lambda, K}: V_{\lambda} \rightarrow V_{\lambda}
$$


be the projection to $V_{\lambda, K}$ with kernel $V_{\lambda}^{K}$.

Proposition 3.3. Let $H=H_{K, \Lambda^{\prime}, J}$ and $G \times G / H=\mathcal{O}$.

(1) For any $\lambda \in \Lambda^{\prime} \cap \Lambda^{+}$, the projection $p_{\lambda, K} \in$ End $V_{\lambda}$ is fixed by $H$.

Thus, the map

$$
\text { End } V_{\lambda} \rightarrow k[G \times G], u \mapsto\left((x, y) \mapsto \operatorname{Tr}_{V_{\lambda}}\left(u x p_{\lambda, K} y^{-1}\right)\right)
$$

is a $G \times G$-equivariant morphism, with image in $k[\mathcal{O}]$.

(2) The resulting map

$$
\bigoplus_{\lambda \in \Lambda^{\prime} \cap \Lambda^{+}} \text {End } V_{\lambda} \rightarrow k[\mathcal{O}]
$$

is an isomorphism of $G \times G$-modules. As a consequence, $\Lambda_{\mathcal{O}}^{+}$identifies to $\Lambda^{\prime} \cap \Lambda^{+}$.

(3) The space $k[\mathcal{O}]^{\operatorname{diag} G}$ of conjugation-invariant functions has a basis consisting of the functions $\chi_{\lambda, K}:(x, y) \mapsto \operatorname{Tr}_{V_{\lambda}}\left(x p_{\lambda, K} y^{-1}\right)$, where $\lambda \in \Lambda^{\prime} \cap \Lambda^{+}$.

(4) The multiplication in $k[\mathcal{O}]$ is given by

$$
f_{\lambda} \cdot f_{\mu}=\sum_{\nu \in \Lambda^{+}, \nu \leq_{K} \lambda+\mu} \operatorname{Tr}_{1}\left(p_{\lambda \mu}^{\nu}\left(f_{\lambda} \otimes f_{\mu}\right)\right)
$$

for any $f_{\lambda} \in$ End $V_{\lambda}$ and $f_{\mu} \in$ End $V_{\mu}$. In particular,

$$
\chi_{\lambda, K} \cdot \chi_{\mu, K}=\sum_{\nu \in \Lambda^{+}, \nu \leq_{K} \lambda+\mu} c_{\lambda \mu}^{\nu} \chi_{\nu, K},
$$

and the product End $V_{\lambda}$. End $V_{\mu}$ is the sum of those End $V_{\nu}$ such that $\nu \leq_{K} \lambda+\mu$ and $c_{\lambda \mu}^{\nu} \neq 0$.

Proof. (1) Clearly, $p_{\lambda, K}$ is fixed by $\left(R_{u}\left(P_{K}\right) \times R_{u}\left(P_{K}^{-}\right)\right) \operatorname{diag} L_{K}$, and hence by $\left(R_{u}\left(P_{I}\right) \times R_{u}\left(P_{I}^{-}\right)\right) \operatorname{diag} L_{K}$ (since $K \subseteq I$ ). Moreover, since $\lambda$ is orthogonal to $J$, it extends to a character of $L_{J}$, and $L_{J}$ acts on $V_{\lambda, K}$ via that character; thus, $H_{J}$ fixes $V_{\lambda, K}$ pointwise. As another consequence, $L_{J}$ stabilizes $V_{\lambda, K}$ and hence $V_{\lambda}^{K}$ as well. It follows that $p_{\lambda, K}$ is fixed by $H_{J} \times H_{J}$. We conclude that $p_{\lambda, K}$ is fixed by $H$.

(2) One has an equivariant isomorphism

$$
\bigoplus_{\lambda, \mu \in \Lambda^{+}} V_{\lambda}^{*} \otimes_{k} V_{\mu} \otimes_{k} V_{\lambda} \otimes_{k} V_{\mu}^{*} \rightarrow k[G \times G]
$$

that maps $u \otimes v$ to the function $(x, y) \mapsto((x, y) v)(u)$; here $u \in V_{\lambda}^{*} \otimes_{k} V_{\mu}$, $v \in V_{\lambda} \otimes_{k} V_{\mu}^{*}=\left(V_{\lambda}^{*} \otimes_{k} V_{\mu}\right)^{*}$, and $(x, y) \in G \times G$. This yields an isomorphism of $G \times G$-modules:

$$
\bigoplus_{\lambda, \mu \in \Lambda^{+}} V_{\lambda}^{*} \otimes_{k} V_{\mu} \otimes_{k}\left(V_{\lambda} \otimes_{k} V_{\mu}^{*}\right)^{H} \rightarrow k[G \times G]^{H}=k[\mathcal{O}] .
$$

Moreover, $\left(V_{\lambda} \otimes_{k} V_{\mu}^{*}\right)^{H}$ is the subset of $\left(H_{J} \times H_{J}\right) \operatorname{diag} L_{K}$-invariants in

$$
\left(V_{\lambda} \otimes_{k} V_{\mu}^{*}\right)^{R_{u}\left(P_{I}\right) \times R_{u}\left(P_{I}^{-}\right)}=V_{\lambda}^{R_{u}\left(P_{I}\right)} \otimes_{k}\left(V_{\mu}^{*}\right)^{R_{u}\left(P_{I}^{-}\right)} .
$$


And $V_{\lambda}^{R_{u}\left(P_{I}\right)}=V_{\lambda, I}$, whereas $\left(V_{\mu}^{*}\right)^{R_{u}\left(P_{I}^{-}\right)}$is the dual of $V_{\mu, I}$ (since both are simple $L_{I}$-modules with lowest weight $-\mu$ ). Thus, we obtain

$$
\left(V_{\lambda} \otimes_{k} V_{\mu}^{*}\right)^{H}=\operatorname{Hom}^{\left(H_{J} \times H_{J}\right) \operatorname{diag} L_{K}}\left(V_{\mu, I}, V_{\lambda, I}\right) .
$$

By Schur's lemma, that space is one-dimensional if both $V_{\lambda, I}$ and $V_{\mu, I}$ are fixed pointwise by $H_{J}$, and are isomorphic as (simple) $L_{K}$-modules. This is equivalent to: $\lambda=\mu$ vanishes on $T^{\prime}$, that is, $\lambda=\mu \in \Lambda^{\prime}$; then $\lambda$ is orthogonal to $J$, and $\left(V_{\lambda} \otimes_{k} V_{\lambda}^{*}\right)^{H}$ is spanned by $p_{\lambda, I}=p_{\lambda, K}$. Otherwise, $\left(V_{\lambda} \otimes_{k} V_{\mu}^{*}\right)^{H}$ is zero. Now the assertion on the structure of $k[\mathcal{O}]$ follows by keeping track of the isomorphisms.

(3) follows readily from (2).

(4) Let $f_{\lambda}, f_{\mu} \in k[\mathcal{O}]$ be the images of $u_{\lambda} \in$ End $V_{\lambda}, u_{\mu} \in$ End $V_{\mu}$, where $\lambda, \mu \in \Lambda^{\prime} \cap \Lambda^{+}$. Then one has for $x, y \in G$ :

$$
\begin{aligned}
& \left(f_{\lambda} \cdot f_{\mu}\right)(x, y)=\operatorname{Tr}_{V_{\lambda} \otimes_{k} V_{\mu}}\left(u_{\lambda} x p_{\lambda, K} y^{-1} \otimes u_{\mu} x p_{\mu, K} y^{-1}\right) \\
& \quad=\operatorname{Tr}_{V_{\lambda} \otimes_{k} V_{\mu}}\left(\left(u_{\lambda} \otimes u_{\mu}\right)(x \otimes x)\left(p_{\lambda, K} \otimes p_{\mu, K}\right)\left(y^{-1} \otimes y^{-1}\right)\right) .
\end{aligned}
$$

Now we claim that

$$
p_{\lambda, K} \otimes p_{\mu, K}=\sum_{\nu \in \Lambda^{+}, \nu \leq_{K} \lambda+\mu} 1 \otimes p_{\nu, K}
$$

in End $V_{\lambda} \otimes_{k}$ End $V_{\mu}=\bigoplus_{\nu}$ End $N_{\lambda \mu}^{\nu} \otimes_{k}$ End $V_{\nu}$. To see this, notice that the subspace

$$
V_{\lambda, K} \otimes_{k} V_{\mu, K} \subseteq V_{\lambda} \otimes_{k} V_{\mu}
$$

is the sum of the $T$-weight spaces associated with those weights $\chi$ such that $\chi \leq_{K} \lambda+\mu$, so that

$$
V_{\lambda, K} \otimes_{k} V_{\mu, K}=\bigoplus_{\nu \in \Lambda^{+}, \nu \leq K}{ }_{\leq^{\prime}+\mu} N_{\lambda \mu}^{\nu} \otimes_{k} V_{\nu, K}
$$

The claim follows, since

$$
p_{\lambda, K} \otimes p_{\mu, K}: V_{\lambda} \otimes_{k} V_{\mu} \rightarrow V_{\lambda} \otimes_{k} V_{\mu}
$$

is the unique $T$-equivariant projection to $V_{\lambda, K} \otimes_{k} V_{\mu, K}$.

Using that claim, we obtain

$$
\left(f_{\lambda} \cdot f_{\mu}\right)(x, y)=\sum_{\nu \in \Lambda^{+}, \nu \leq_{K} \lambda+\mu} \operatorname{Tr}_{N_{\lambda \mu}^{\nu} \otimes_{k} V_{\nu}}\left(p_{\lambda \mu}^{\nu}\left(u_{\lambda} \otimes u_{\mu}\right)\left(1 \otimes x p_{\nu, K} y^{-1}\right)\right)
$$

which implies our assertions.

Next we characterize those homogeneous spaces $G \times G / H_{K, \Lambda^{\prime}, J}$ that are quasi-affine, and we describe their $\operatorname{diag} T$-fixed points.

Proposition 3.4. Let $H=H_{K, \Lambda^{\prime}, J}$ and $G \times G / H=\mathcal{O}$. Then $\mathcal{O}$ is quasiaffine if and only if: $\Lambda^{\prime} \cap \Lambda^{+}$spans $\Lambda^{\prime}$, and $J$ is the set of those simple roots that are orthogonal to $\Lambda^{\prime}$. 
In that case, one has $\Lambda_{\mathcal{O}}=\Lambda^{\prime}$, and $\mathcal{O}^{\operatorname{diag} T}=(\operatorname{diag} W) \mathcal{O}_{0}^{\operatorname{diag} T}$, where $\mathcal{O}_{0}^{\operatorname{diag} T}$ is the $T \times T$-orbit $(T \times T) H / H \simeq T / T^{\prime}$. Moreover, the subgroup of $W$ that fixes $\mathcal{O}_{0}^{\operatorname{diag} T}$ pointwise (resp. stabilizes $\mathcal{O}_{0}^{\operatorname{diag} T}$ ) is $W_{J}\left(\right.$ resp. $\left.W_{J} \times W_{K}\right)$.

Finally, $(\operatorname{diag} G) \mathcal{O}^{\operatorname{diag} T}$ is dense in $\mathcal{O}$.

Proof. Since the monoid $\Lambda^{\prime} \cap \Lambda^{+}$is finitely generated, the algebra $k[\mathcal{O}]$ is finitely generated as well (this follows e.g. from Gro97 Theorem 16.2). Let $X$ be the corresponding affine irreducible $G \times G$-variety, then one has a dominant morphism $\mathcal{O} \rightarrow X$ with irreducible general fibers, since $k[\mathcal{O}]=$ $k[G \times G]^{H}$ is integrally closed in the function field $k(\mathcal{O})=k(G \times G)^{H}$. Thus, $G \times G / H$ is quasi-affine if and only if $\operatorname{dim} G \times G / H=\operatorname{dim} X$.

Now $\operatorname{dim} X=\operatorname{rank}\left(\Lambda^{\prime} \cap \Lambda^{+}\right)+\left|\Phi-\left(\Lambda^{\prime} \cap \Lambda^{+}\right)^{\perp}\right|$, where $\left(\Lambda^{\prime} \cap \Lambda^{+}\right)^{\perp}$ denotes the set of those roots that are orthogonal to $\Lambda^{\prime} \cap \Lambda^{+}$(as follows e.g. from Weyl's dimension formula). Then $\left(\Lambda^{\prime} \cap \Lambda^{+}\right)^{\perp}=\Phi_{J^{\prime}}$ for some $J^{\prime} \supseteq J$. So $\operatorname{dim} \mathcal{O}=\operatorname{dim} X$ if and only if: $\Lambda^{\prime} \cap \Lambda^{+}$spans $\Lambda^{\prime}$, and $J^{\prime}=J$. This proves the first assertion.

For the second assertion, let $(x, y) \in G \times G$ such that the coset $(x, y) H$ is in $\mathcal{O}^{\operatorname{diag} T}$. We claim that $(x, y) \in \operatorname{diag} N(T \times T) H$. Note that

$$
\left(n t_{1} x h_{1}, n t_{2} y h_{2}\right) \in \mathcal{O}^{\operatorname{diag} T}
$$

for all $n \in N,\left(t_{1}, t_{2}\right) \in T \times T$ and $\left(h_{1}, h_{2}\right) \in H$. Moreover, since $H \subseteq$ $P_{I} \times P_{I}^{-}$, the coset $\left(x P_{I}, y P_{I}^{-}\right)$is a $\operatorname{diag} T$-fixed point in $G / P_{I} \times G / P_{I}^{-}$. Thus, $x \in N P_{I}$ and $y \in N P_{I}^{-}$. Since $P_{I}^{-}$is the image of $H$ under the second projection to $G$, we may assume that $y=1$. Moreover, replacing $x$ by $x h_{1}$ where $h_{1} \in H \cap(G \times 1)=R_{u}\left(P_{I}\right) H_{J}$, we may assume that $x \in N L_{K}$. Then $\left(x^{-1} t x, t\right) \in L_{K} \times T$ for all $t \in T$. On the other hand, $\left(x^{-1} t x, t\right) \in H$ since $\operatorname{diag} T$ fixes $(x, 1) H$. Thus,

$$
\left(x^{-1} t x, t\right) \in H \cap\left(L_{K} \times T\right)=\left(T^{\prime} \times T^{\prime}\right) \operatorname{diag} T .
$$

In particular, $x^{-1} t x \in T$ for all $t \in T$, that is, $x \in N$. And $x^{-1} t x t^{-1} \in T^{\prime}$, so that $\chi\left(x^{-1} t x\right)=\chi(t)$ for all $\chi \in \Lambda^{\prime}$ : in other words, $x$ fixes $\Lambda^{\prime}$ pointwise. Thus, $x$ fixes pointwise $\Lambda^{\prime} \cap \Lambda^{+}$, so that $x \in N \cap L_{J}$. Hence $x \in T(H \cap(G \times 1))$, so that $(x, 1) \in(T \times T) H$. This completes the proof of the claim.

By that claim, one has $\mathcal{O}^{\operatorname{diag} T}=(\operatorname{diag} W) \mathcal{O}_{0}^{\operatorname{diag} T}$. Moreover, a given $w=n T \in W$ stabilizes $\mathcal{O}_{0}^{\operatorname{diag} T}$ if and only if $(n, n) \in(T \times T) H$, that is, $w \in W_{J} \times W_{K}$. Finally, $w$ fixes $\mathcal{O}_{0}^{\operatorname{diag} T}$ pointwise if and only if it fixes $\Lambda^{\prime}$ pointwise, that is, $w \in W_{J}$.

For the final assertion, note that $(\operatorname{diag} G) \mathcal{O}^{\operatorname{diag} T}=(\operatorname{diag} G) \mathcal{O}_{0}^{\operatorname{diag} T}$ is dense in $\mathcal{O}$ if and only if the subset $(\operatorname{diag} G)(T \times T) H$ is dense in $G \times G$, or (quotienting by $\operatorname{diag} G$ ): the subset

$$
\left\{x t y^{-1} \mid(x, y) \in H, t \in T\right\}
$$

is dense in $G$. Since the product $R_{u}\left(P_{I}\right) L_{I} R_{u}\left(P_{I}^{-}\right)$is open in $G$, it suffices to show that the subset

$$
\left\{x t y^{-1} \mid(x, y) \in H \cap\left(L_{I} \times L_{I}\right), t \in T\right\}
$$


is dense in $L_{I}$. Since $L_{I}=\left[L_{J}, L_{J}\right] L_{K}$ and $H \cap\left(L_{I} \times L_{I}\right)=\left(H_{J} \times H_{J}\right) \operatorname{diag} L_{K}$ where $H_{J}$ contains $\left[L_{J}, L_{J}\right]$, this reduces to the well-known fact that the subset $\left\{h t h^{-1} \mid h \in L_{K}, t \in T\right\}$ is dense in $L_{K}$.

Finally, we record the following easy result on the multiplication in the ring $k\left[G \times G / H_{K, \Lambda^{\prime}, J}\right]$.

Lemma 3.5. Let $H=H_{K, \Lambda^{\prime}, J}$ be such that $\mathcal{O}=G \times G / H$ is quasi-affine. Then, for any $\alpha \in K$, there exists $\lambda \in \Lambda^{\prime} \cap \Lambda^{+}$such that $\langle\lambda, \check{\alpha}\rangle \neq 0$. In that case, $2 \lambda-\alpha \in \Lambda^{+}$, and the product End $V_{\lambda} \cdot$ End $V_{\lambda} \subset k[\mathcal{O}]$ contains a unique copy of the $G \times G$-module End $V_{2 \lambda-\alpha}$. A corresponding $B^{-} \times B$-eigenvector is

$$
\left(\eta_{\lambda} \otimes v_{\lambda}\right) \cdot\left(e_{\alpha} \eta_{\lambda} \otimes e_{-\alpha} v_{\lambda}\right)-\left(e_{\alpha} \eta_{\lambda} \otimes v_{\lambda}\right) \cdot\left(\eta_{\lambda} \otimes e_{-\alpha} v_{\lambda}\right),
$$

where $\eta_{\lambda}$ (resp. $v_{\lambda}$ ) is a lowest (resp. highest) weight vector in $V_{\lambda}^{*}$ (resp. $V_{\lambda}$ ), and $e_{ \pm \alpha}$ is a root vector in $\operatorname{Lie}(G)$ of weight $\pm \alpha$.

Proof. If $\check{\alpha}$ is orthogonal to $\Lambda^{\prime} \cap \Lambda^{+}$, then it is orthogonal to $\Lambda^{\prime}$ by Proposition 3.4. But this contradicts the assumption that $\Lambda^{\prime}$ contains $K$. This proves the first assertion.

For any $\lambda \in \Lambda^{+}$and $\alpha \in \Pi$ such that $\langle\lambda, \check{\alpha}\rangle \neq 0$, the weight $2 \lambda-\alpha$ is dominant, and the corresponding simple module $V_{2 \lambda-\alpha}$ occurs in $V_{\lambda} \otimes_{k} V_{\lambda}$ with multiplicity 1 , a corresponding highest weight vector being $v_{\lambda} \otimes e_{-\alpha} v_{\lambda}-$ $e_{-\alpha} v_{\lambda} \otimes v_{\lambda}$. This implies the remaining assertions.

\section{Reductive VARIETIES}

4.1. Classification. From the combinatorial classification of the group-like homogeneous spaces in Section 3 we will deduce a similar classification of reductive varieties. We begin with a characterization of these varieties in terms of their weight set.

Proposition 4.1. For an affine irreducible $G \times G$-variety $X$, the following conditions are equivalent:

(1) $X$ is a reductive variety.

(2) $X$ is diagonal, multiplicity-free, and $\Lambda_{X}^{+}$is saturated in $\Lambda$.

Then all $G \times G$-orbit closures in $X$ are normal.

Proof. (1) $\Rightarrow(2)$ By Proposition $2.7 X$ is diagonal and multiplicity-free. And since the isotropy groups of $X$ are connected, $\Lambda_{X}$ is saturated in $\Lambda$ by Lemma 3.2 and Proposition 3.4. Together with Lemmas 2.2] and 2.5] it follows that all $G \times G$-orbit closures in $X$ are normal, and that $\Lambda_{X}^{+}=\Lambda \cap C_{X}$.

$(2) \Rightarrow(1)$ follows from Propositions 2.4 and 2.7

Next we construct all reductive varieties. For any subset $K \subseteq \Pi$, consider the closed subgroup of $G \times G$ :

$$
H_{K}=H_{K, \Lambda, \emptyset}=\left(R_{u}\left(P_{K}\right) \times R_{u}\left(P_{K}^{-}\right)\right) \operatorname{diag} L_{K} .
$$


Let $k[G]_{(K)}$ be the algebra of regular functions on the homogeneous space $G \times G / H_{K}$. Then $k[G]_{(K)}=\bigoplus_{\lambda \in \Lambda^{+}}$End $V_{\lambda}$ as a $G \times G$-module; its multiplication is given by

$$
f_{\lambda} \cdot f_{\mu}=\sum_{\nu \in \Lambda^{+}, \nu \leq_{K} \lambda+\mu} \operatorname{Tr}_{1}\left(p_{\lambda \mu}^{\nu}\left(f_{\lambda} \otimes f_{\mu}\right)\right) .
$$

Thus, we may regard the algebra $k[G]_{(K)}$ as a degeneration of $k[G]$; this will be developed in Subsection [7.5 below.

Proposition 4.2. Let $K$ be a subset of $\Pi$ and let $C$ be a cone in $\Lambda_{\mathbb{R}}^{+}$, satisfying the following conditions:

(1) The linear span $\operatorname{lin} C$ contains $K$.

(2) $C$ is the intersection of $\operatorname{lin} C \cap \Lambda_{\mathbb{R}}^{+}$with a finite (possibly empty) family $\left(v_{i} \geq 0\right)_{i \in I}$ of closed half-spaces of $\Lambda_{\mathbb{R}}$, where $v_{i} \leq 0$ on $K$ for all $i$.

Then $C$ is a rational polyhedral convex cone; the $G \times G$-invariant subspace

$$
k[G]_{(C, K)}=\bigoplus_{\lambda \in \Lambda \cap C} \text { End } V_{\lambda}
$$

of $k[G]_{(K)}$ is a finitely generated subalgebra, and the corresponding affine $G \times G$-variety $X_{C, K}$ is a reductive variety for $G$. It is equipped with a base point $x_{C, K}$ in its open $B^{-} \times B$-orbit, fixed by $\operatorname{diag} T$.

Conversely, each reductive variety is isomorphic to some $X_{C, K}$, where $C, K$ are uniquely determined and satisfy (1) and (2). Moreover, the isotropy group of $x_{C, K}$ is $H_{K, \Lambda^{\prime}, J}$, where $\Lambda^{\prime}$ is the subgroup of $\Lambda$ generated by $\Lambda \cap C$, and $J$ is the set of simple roots orthogonal to $C$. Finally, Aut ${ }^{G \times G}\left(X_{C, K}\right)$ is a diagonalizable group with character group $(\Lambda \cap \operatorname{lin} C) / \mathbb{Z} K$.

Proof. This follows from embedding theory of spherical homogeneous spaces (see $[$ Kno89 $)$. We give a direct proof for the reader's convenience.

The multiplication in $k[G]_{(K)}$ satisfies

$$
\text { End } V_{\lambda} \cdot \text { End } V_{\mu} \subseteq \bigoplus_{\nu \leq K} \bigoplus_{+\mu} \text { End } V_{\nu} .
$$

Together with condition (2), it follows that $k[G]_{(C, K)}$ is a subalgebra of $k[G]_{(K)}$. Its subalgebra $k[G]_{(C, K)}^{U^{-} \times U}$ is isomorphic to the algebra of the monoid $\Lambda \cap C$ over $k$. This monoid is finitely generated by Gordan's lemma; hence the same holds for $k[G]_{(C, K)}^{U^{-} \times U}$, and for $k[G]_{(C, K)}$ by Gro97] Theorem 16.2. Now $X_{C, K}$ is a reductive variety by Proposition 4.1. It is equipped with a dominant $G \times G$-equivariant morphism $G \times G / H_{K} \rightarrow X_{C, K}$ and hence with a base point $x_{C, K}$ (the image of the coset $H_{K}$ ).

Conversely, let $X$ be a reductive variety. Then $X$ is normal by Proposition 4.1 again; and by Propositions 3.1 and 3.4 $X$ contains an open $G \times G$-orbit isomorphic to $G \times G / H$ for some $H=H_{K, \Lambda^{\prime}, J}$, where $J$ is the set of simple 
roots orthogonal to $\Lambda^{\prime}$. Then $H \subseteq H_{K}$ so that $k[X]$ identifies to a $G \times G$ invariant subalgebra of $k[G]_{(K)}$. Since $X$ is normal, we have by Lemmas 2.1 and 2.2 $k[X]=\bigoplus_{\lambda \in \Lambda \cap C}$ End $V_{\lambda}$ for a uniquely defined rational polyhedral convex cone $C$ in $\Lambda_{\mathbb{R}}^{+}$. Moreover, $\Lambda \cap C$ spans $\Lambda^{\prime}$ (since $k[X]$ and $k[G \times G / H]$ have the same fraction field), so that $\operatorname{lin} C$ contains $K$. Let $\left(X_{i}\right)_{i \in I}$ be the (finite, possibly empty) set of closed irreducible $G \times G$-subvarieties of codimension 1 in $X$, and let $\left(v_{i}\right)_{i \in I}$ be the corresponding set of valuations of the field of rational functions $k(X)=k(G \times G / H)$. Then each valuation ring $\mathcal{O}_{v_{i}}$ is stable by $G \times G$, and

$$
k[X]=k[G \times G / H] \cap \bigcap_{i \in I} \mathcal{O}_{v_{i}} .
$$

Moreover, for any $T \times T$-eigenvector $f \in k[X]^{U^{-} \times U}$ with weight $\lambda$, the value $v_{i}(f)$ depends only on $\lambda$ (since $\lambda$ determines uniquely $f$ up to scalar). This defines an additive map $v_{i}: \Lambda_{X}^{+} \rightarrow \mathbb{Z}$; it extends to a linear functional on $\Lambda_{\mathbb{R}}$ that we still denote by $v_{i}$. Then

$$
\Lambda_{X}^{+}=\Lambda_{G \times G / H} \cap \bigcap_{i \in I}\left(v_{i} \geq 0\right) .
$$

Finally, since $v_{i}$ is a $G \times G$-invariant valuation, it is constant on every End $V_{\lambda}-\{0\}$, with value $v_{i}(\lambda)$. And since

$$
\text { End } V_{2 \lambda-\alpha} \subset \text { End } V_{\lambda} \cdot \text { End } V_{\lambda}
$$

for any $\alpha \in \Pi$ such that $\langle\lambda, \check{\alpha}\rangle>0$ (Lemma 3.5), it follows that $v_{i}(\alpha) \leq 0$ for any $\alpha \in K$.

Recall from Lemma 3.2 that $\operatorname{Aut}^{G \times G}(G \times G / H)=Z_{K} / T_{H}$ is diagonalizable with character group $\Lambda^{\prime} / \mathbb{Z} K$. Moreover, Aut ${ }^{G \times G}(G \times G / H)$ acts by scalars on every subspace End $V_{\lambda}$ of $k[G \times G / H]$ (the corresponding character being $\lambda \in \Lambda^{\prime}$.) Thus, Aut ${ }^{G \times G}(G \times G / H)$ stabilizes the subalgebra $k[X]$; this yields an isomorphism $\operatorname{Aut}^{G \times G}(X) \simeq$ Aut $^{G \times G}(G \times G / H)$.

4.2. Associated stable toric varieties. We will describe the subsets of $\operatorname{diag} T$-fixed points in reductive varieties for $G$. A useful preliminary result is the following:

Lemma 4.3. Let $X$ be a stable reductive variety for $G$. Then $X$ contains a unique closed $G \times G$-orbit $Y$. The latter is isomorphic to $G / G_{1}$, where $G_{1}$ is a closed connected normal subgroup of $G$ (and $G \times G$ acts on $G / G_{1}$ by left and right multiplication). In particular, $Y^{\operatorname{diag} T}$ is a unique $T \times T$-orbit.

Moreover, there exists a stable reductive variety $X_{1}$ (for $G_{1}$ ) with a fixed point of $G_{1} \times G_{1}$, such that $X \simeq G \times{ }^{G_{1}} X_{1}$ (the quotient of $G \times X_{1}$ by the $G_{1}$-action: $g_{1} \cdot(g, x)=\left(g g_{1}^{-1}, g_{1} x\right)$.)

Proof. Since $X$ is connected and contains only finitely many $G \times G$-orbits, $X / / G$ is connected and finite. Thus, $X$ contains a unique closed orbit $Y=$ $G \times G / H$; then $H$ is connected and reductive. By Proposition 3.1, it follows that $H=\left(H_{J} \times H_{J}\right) \operatorname{diag} L_{K}$, where $\Pi$ is the union of orthogonal subsets $J$ 
and $K$, and $\left[L_{J}, L_{J}\right] \subseteq H_{J} \subseteq\left[L_{J}, L_{J}\right] Z_{K}$. Thus, $H_{J}$ is a normal subgroup of $G$, and $H_{J} \times H_{J}$ fixes pointwise $G \times G / H$. Replacing $G$ by $G / H_{J}$, we may assume that $H_{J}$ is trivial; then $L_{K}=G$, so that $Y \simeq G$. This proves the first assertion; the second assertion then follows from Luna's slice theorem.

Proposition 4.4. Let $X=X_{C, K}$ be a reductive variety for $G$, with open $B^{-} \times B$-orbit $X_{0}$. Let $X^{\prime}$ be the closure of $X_{0}^{\operatorname{diag} T}$ in $X$. Then:

(1) $X^{\prime}$ is a toric variety for $T$, with lattice $\Lambda_{X}$ and cone $W_{K} C$. In particular, $X^{\prime}$ is normal, and $\sigma=W_{K} C$ is a rational polyhedral convex cone. Moreover, one has

$\sigma \cap \Lambda_{\mathbb{R}}^{+}=C$, and $K=\left\{\alpha \in \Pi \mid \sigma^{0}\right.$ meets the hyperplane $\left.(\alpha=0)\right\}$, where $\sigma^{0}$ is the relative interior of $\sigma$.

(2) $X^{\operatorname{diag} T}=(\operatorname{diag} W) X^{\prime}$ is a diagonal, multiplicity-free T-variety with weight set $\Lambda \cap W \sigma$. As a consequence, $X^{\operatorname{diag} T}$ is a stable toric variety, and the distinct translates $w \sigma^{0}(w \in W)$ are disjoint. Moreover, $X^{\operatorname{diag} T}$ meets any $G \times G$-orbit along a unique $W T$-orbit.

Proof. (1) Let $x=x_{C, K} \in X_{C, K}$ be the base point, with isotropy group $H$; then $x \in X_{0}^{\operatorname{diag} T}$. Thus, $X^{\prime}$ is the closure of $(T \times T) x$, so that we may identify $k\left[X^{\prime}\right]$ with a $T \times T$-invariant subalgebra of $k[T \times T / \operatorname{diag} T] \simeq k[T]$. On the other hand, the vector space $k\left[X^{\prime}\right]$ is spanned by the restrictions to $X^{\prime}$ of all subspaces End $V_{\lambda}$ of $k[X]$. By Proposition 3.3. it follows that $k\left[X^{\prime}\right]$ is spanned by the functions on $T \times T$ :

$$
(x, y) \mapsto \operatorname{Tr}_{V_{\lambda}}\left(u x p_{\lambda, K} y^{-1}\right)=\operatorname{Tr}_{V_{\lambda, K}}\left(p_{\lambda, K} y^{-1} u x\right)
$$

where $u \in$ End $V_{\lambda}$. So the weights of End $\left.V_{\lambda}\right|_{X^{\prime}}$ are precisely the weights of the $T$-module $V_{\lambda, K}$, that is, those weights $\chi \in \operatorname{Conv}\left(W_{K} \lambda\right)$ that satisfy $\chi \leq_{K} \lambda$. This set of weights is $W_{K}$-invariant, and for any such weight $\chi$, there exists $w \in W_{K}$ such that $\langle w \chi, \check{\alpha}\rangle \geq 0$ for all $\alpha \in K$. On the other hand, since $\lambda$ is dominant and $w \chi \leq_{K} \lambda$, one has $\langle w \chi, \check{\alpha}\rangle \geq 0$ for all $\alpha \in \Pi-K$. So $w \chi \in \Lambda_{\mathbb{R}}^{+}$, whence $w \chi \in \Lambda \cap C$ by condition (2) of Proposition 4.2 Thus, $\Lambda_{X^{\prime}}^{+} \subseteq W_{K}(\Lambda \cap C)$, and the opposite inclusion is obvious: we have proved that $\Lambda_{X^{\prime}}^{+}=W_{K}(\Lambda \cap C)$.

It follows that $\Lambda_{X^{\prime}}=\Lambda_{X}$ and that $\Lambda_{X^{\prime}}^{+}$is saturated in this subgroup. Since $X^{\prime}$ is irreducible, it is a toric variety with cone $W_{K} C=\sigma$. Moreover, the argument shows that $\sigma \cap \Lambda_{\mathbb{R}}^{+}=C$.

Let $\alpha \in \Pi$. If $\alpha \notin K$, then $w \alpha \in \Phi^{+}$for all $w \in W_{K}$. Thus, $\alpha \geq 0$ on $W_{K} \Lambda_{\mathbb{R}}^{+}$, and hence on $\sigma$. Conversely, if $\alpha \in K$, then $\alpha$ takes positive values on $C$ (otherwise $\alpha$ is orthogonal to $C$, which contradicts the assumption that $\alpha \in \operatorname{lin} C$ ). Thus, $\alpha$ takes negative values on $\sigma=W_{K} C$, so that $C$ meets the hyperplane $(\alpha=0)$. This completes the proof of (1).

(2) By Proposition 3.4 $X^{\operatorname{diag} T}$ meets any $G \times G$-orbit $\mathcal{O}$ along a unique $W T$-orbit. On the other hand, we assert that $X^{\prime}$ meets any $G \times G$-orbit. This may be deduced from embedding theory of spherical homogeneous spaces (see [Kno89]); here is a direct argument, using the notation of Proposition 
3.1. Since $P_{I} \times P_{I}^{-}$is a parabolic subgroup of $G \times G$, the closure of $\left(P_{I} \times P_{I}^{-}\right) x$ in $X$ meets all $G \times G$-orbits. Moreover,

$$
\left(P_{I} \times P_{I}^{-}\right) x \simeq\left(P_{I} \times P_{I}^{-}\right) H / H \simeq\left(L_{I} \times L_{I}\right) /\left(L_{I} \times L_{I}\right) \cap H
$$

is isomorphic (as a $L_{I} \times L_{I}$-variety) to the quotient $L_{I} / H_{J}$ where $L_{I}$ acts by left and right multiplication. This quotient is a connected reductive group $G_{1}$, and the image of $T$ is a maximal torus $T_{1}$. Now the closure of $T_{1}$ in any $G_{1} \times G_{1}$-equivariant embedding of $G_{1}$ meets all $G_{1} \times G_{1}$-orbits, as follows e.g. from a theorem of Iwahori (see [Mum94, p. 52).

It follows that the irreducible components of $X^{\operatorname{diag} T}$ are the $w X^{\prime}(w \in W)$, and also (using Lemma 4.3) that $X^{\operatorname{diag} T}$ is connected. Thus, the weight set of $X^{\operatorname{diag} T}$ is $W(\Lambda \cap C)=\Lambda \cap W \sigma$. We now claim that $X^{\operatorname{diag} T}$ is multiplicityfree. It suffices to check that the multiplicity of any $\lambda \in \Lambda \cap C$ is 1 . Let $f$ be a $T \times T$-eigenvector in $k[X]^{U^{-} \times U}$ of weight $\lambda$, then $\left.f\right|_{X^{\prime}}$ is a $T \times T$-eigenvector of weight $\lambda$, which does not vanish identically on any $w X^{\prime}, w \in \operatorname{Stab}_{W}(\lambda)$. Let $\varphi \in k\left[X^{\prime}\right]$ be another $T \times T$-eigenvector of weight $\lambda$. If $\varphi$ does not vanish identically on $w X^{\prime}$, then $w^{-1}(\lambda) \in W_{K} C$, whence $w \in \operatorname{Stab}_{W}(\lambda) W_{K}$, and $w X^{\prime} \subseteq \operatorname{Stab}_{W}(\lambda) X^{\prime}$. So it suffices to show that $\varphi$ is a scalar multiple of $f$ when restricted to $\bigcup_{w \in \operatorname{Stab}_{W}(\lambda)} w X_{0}^{\operatorname{diag} T}$, a subset of $X_{f}^{\operatorname{diag} T}$. With the notation of Lemma 2.8 $X_{f}^{\operatorname{diag} T}=Z^{\operatorname{diag} T}$; the latter is connected and contains only finitely many $T \times T$-orbits. Thus, the quotient $\varphi / f$, a regular $T \times T$-invariant function on $Z^{\operatorname{diag} T}$, is constant. This proves our claim.

Now $X^{\operatorname{diag} T}$ is a stable toric variety, by the claim and Lemma 2.3. And since it is multiplicity-free, the distinct $w \sigma^{0}$ are disjoint.

Proposition 4.5. For any reductive variety $X$, the natural morphism

$$
p: X^{\operatorname{diag} T} / \operatorname{diag} W \rightarrow X / / \operatorname{diag} G
$$

is an isomorphism.

Moreover, the closed $\operatorname{diag} G$-orbits in $X$ are exactly the orbits of points in $X^{\operatorname{diag} T}$; the union of these orbits contains a dense subset of every $G \times G$ orbit.

Finally, $X$ is self-adjoint for a unique automorphism $\Theta_{X}$ fixing pointwise $X^{\operatorname{diag} T}$.

Proof. By the main result of Lun75, the morphism $p$ is finite; on the other hand, it is well-known that the orbit $(\operatorname{diag} G) x$ is closed for every $x \in X^{\operatorname{diag} T}$, and that its $T$-fixed point subset is just ( $\operatorname{diag} W) x$. Moreover, by Proposition 3.4, the set ( $\operatorname{diag} G) X^{\operatorname{diag} T}$ contains a dense open subset of every $G \times G$-orbit. As a consequence, $p$ is birational. Since $X$ is normal, $p$ is an isomorphism. In particular, it is surjective, so that every closed $\operatorname{diag} G$-orbit meets $X^{\operatorname{diag} T}$.

By Lemma 3.2. the open orbit $G \times G / H$ is self-adjoint for an automorphism $\Theta_{G \times G / H}$ fixing pointwise $(T \times T) H / H$. Since $\Theta\left(\right.$ End $\left.V_{\lambda}\right) \simeq$ End $V_{\lambda}$ as $G \times G$-modules, $\Theta_{G \times G / H}$ stabilizes each simple submodule of $k[G \times G / H]$. Hence it stabilizes the subalgebra $k[X]$; this yields an automorphism $\Theta_{X}$ 
making $X$ self-adjoint. Since $(\operatorname{diag} W)(T \times T) H / H$ is dense in $X^{\operatorname{diag} T}$, and $\Theta$ fixes $\operatorname{diag} W$ pointwise, it follows that $\Theta_{X}$ fixes pointwise $X^{\operatorname{diag} T}$. Thus,

$$
\Theta_{X}((g, g) x)=(\theta(g), \theta(g)) x
$$

for any $g \in G$ and $x \in X^{\operatorname{diag} T}$. This implies uniqueness of $\Theta_{X}$, since $(\operatorname{diag} G) X^{\operatorname{diag} T}$ is dense in $X$.

Next we reformulate the combinatorial classification of reductive varieties by pairs $(C, K)$, in terms of the unique datum $W_{K} C=\sigma$. This makes sense in view of Proposition 4.4 which motivates the following

Definition 4.6. A $W$-admissible cone is a rational polyhedral convex cone $\sigma$ in $\Lambda_{\mathbb{R}}$, satisfying the following conditions:

(1) The relative interior $\sigma^{0}$ meets $\Lambda_{\mathbb{R}}^{+}$.

(2) The distinct $w \sigma^{0}(w \in W)$ are disjoint.

Thus, if $C, K$ satisfy the conditions of Proposition 4.2, then $\sigma=W_{K} C$ is a $W$-admissible cone. Conversely, we have the following easy result.

Lemma 4.7. Let $\sigma$ be a $W$-admissible cone. Let $C=C_{\sigma}=\sigma \cap \Lambda_{\mathbb{R}}^{+}$and $K=K_{\sigma}=\left\{\alpha \in \Pi \mid(\alpha=0)\right.$ meets $\left.\sigma^{0}\right\}$. Then $C, K$ satisfy conditions (1), (2) of Proposition 4.2.

Proof. Let $\alpha \in K$. Then $s_{\alpha} \sigma^{0}$ meets $\sigma^{0}$. Thus, $s_{\alpha} \sigma^{0}=\sigma^{0}$, and $\sigma$ is invariant under $s_{\alpha}$. So $\sigma$ is invariant under $W_{K}$, and also $K \subset \operatorname{lin} \sigma=\operatorname{lin} C$. Hence condition (1) holds. Moreover, $\sigma$ is the intersection of finitely many closed half-spaces $\left(v_{i} \geq 0\right)_{i \in I}$, together with all their $W_{K}$-translates. Moving $v_{i}$ in its $W_{K}$-orbit, we may assume that $v_{i} \leq 0$ on $K$ for any $i \in I$.

We claim that $\sigma=W_{K} C$. Let $\tau=\sigma \cap(\alpha \geq 0)$, then $\sigma=\tau \cup s_{\alpha} \tau$, and $\tau^{0} \cap s_{\alpha} \tau^{0}=\emptyset$. It follows that $\tau$ is a $W$-admissible cone, such that $C_{\tau}=C_{\sigma}$ and $K_{\tau} \subseteq K_{\sigma}-\{\alpha\}$. By induction, we thus obtain $\sigma \subseteq W_{K} C_{\sigma}$. The opposite inclusion is obvious; this proves the claim.

This claim implies that $C=\operatorname{lin} C \cap \Lambda_{\mathbb{R}}^{+} \cap \bigcap_{i \in I}\left(v_{i} \geq 0\right)$; hence condition (2) holds.

We may now reparametrize the reductive varieties for $G$ in terms of $W$ admissible cones, by setting

$$
X_{\sigma}=X_{C_{\sigma}, K_{\sigma}}
$$

Then $X_{\sigma}^{\operatorname{diag} T}$ is the stable toric variety associated with the complex of cones in $\Lambda_{\mathbb{R}}$ consisting of all translates $w \sigma$ and their faces; these are in bijection with the $T$-orbits in $X_{\sigma}^{\operatorname{diag} T}$ (see [Ale02 Corollary 2.3.9). Thus, the $W T$ orbits in $X_{\sigma}^{\operatorname{diag} T}$ are in bijection with the $W$-orbits of faces of $w \sigma, w \in W$. Moreover, the restriction map

$$
\operatorname{Aut}^{G \times G}\left(X_{\sigma}\right) \rightarrow \operatorname{Aut}^{W T}\left(X_{\sigma}^{\operatorname{diag} T}\right)
$$

is an isomorphism. 
Indeed, let $X=X_{\sigma}, K=K_{\sigma}$ and let $X^{\prime}$ be as in Proposition 4.4. Then $X^{\operatorname{diag} T}=W X^{\prime}$, and $\operatorname{Stab}_{W}\left(X^{\prime}\right)=\operatorname{Stab}_{W}(\sigma)$ acts on it through its quotient $W_{K}$, so that $\mathrm{Aut}^{W T}\left(X^{\operatorname{diag} T}\right)$ injects into $\mathrm{Aut}^{W_{K} T}\left(X^{\prime}\right)$. Moreover, $\operatorname{Aut}^{T}\left(X^{\prime}\right)$ is the quotient of $T$ with character group $\Lambda \cap \operatorname{lin} \sigma$, and $\operatorname{Aut}^{W_{K} T}\left(X^{\prime}\right)$ is its subgroup with character group $\Lambda \cap \operatorname{lin} \sigma / \mathbb{Z} K$. By Proposition 4.2 it follows that $\mathrm{Aut}^{G \times G}\left(X_{\sigma}\right) \rightarrow \mathrm{Aut}^{W_{K} T}\left(X^{\prime}\right)$ is an isomorphism.

So we have proved the following "toric correspondence":

Theorem 4.8. The assignment $X \mapsto X^{\operatorname{diag} T}$ defines a bijection from the reductive varieties for $G$, to the stable toric varieties for $T$ with a compatible $W$-action such that the quotient by $W$ is irreducible. Moreover, the $G \times G$ orbits in $X$ are in bijection with the WT-orbits in $X^{\operatorname{diag} T}$, and $\mathrm{Aut}^{G \times G}(X)$ is isomorphic to the automorphism group of the WT-variety $X^{\operatorname{diag} T}$.

\section{Stable Reductive VARieties}

We show how to obtain all stable reductive varieties, by glueing reductive varieties along invariant closed subvarieties. For this, we introduce a combinatorial object, that will encode part of the glueing data.

Definition 5.1. A $W$-complex of cones $\Sigma$ referenced by $\Lambda$ is a topological space $|\Sigma|$ represented as a finite union of distinct closed subsets $\sigma(\sigma \in \Sigma)$, together with a map $\rho:|\Sigma| \rightarrow \Lambda_{\mathbb{R}}$ such that:

(1) $\rho$ identifies each $\sigma \in \Sigma$ with a rational polyhedral convex cone in $\Lambda_{\mathbb{R}}$.

(2) If $\sigma \in \Sigma$, then each face $\tau \prec \sigma$ is in $\Sigma$.

(3) If $\sigma, \tau$ in $\Sigma$, then their intersection in $|\Sigma|$ is a union of faces of both.

(4) $W$ acts on $|\Sigma|$, the reference map $\rho$ is $W$-equivariant, and its restriction to any subset $\bigcup_{w \in W} w \sigma$ is injective.

(In particular, $W$ permutes the subsets $\sigma$.)

For example, any $W$-admissible cone $\sigma$ defines a $W$-complex of cones $\Sigma$, as follows: the cones in $\Sigma$ are the $W$-translates of faces of $\sigma$, and the map $\rho:|\Sigma| \rightarrow \Lambda_{\mathbb{R}}$ is just the inclusion $\bigcup_{w \in W} w \sigma \subseteq \Lambda_{\mathbb{R}}$. Such a $W$-complex of cones will be called elementary. Clearly, a $W$-complex of cones is elementary if and only if it admits a unique maximal cone modulo $W$.

Now consider an arbitrary $W$-complex of cones $\Sigma$, and the orbit space $\Sigma / W$. Note that any $\bar{\sigma} \in \Sigma / W$ admits a unique representative $\sigma \in \Sigma$ such that $\rho(\sigma)^{0}$ meets $\Lambda_{\mathbb{R}}^{+}$. This yields a partial ordering $\leq$on $\Sigma / W$, where $\bar{\tau}=W \tau \leq W \sigma=\bar{\sigma}$ if and only if $\tau \prec \sigma$.

Every $\bar{\sigma} \in \Sigma / W$ defines a reductive variety $X_{\bar{\sigma}}=X_{\sigma}$. Moreover, $\bar{\tau} \leq \bar{\sigma}$ if and only if $\tau \prec \sigma$. In that case, by the results of Subsection 4.2 we have a closed $G \times G$-equivariant immersion

$$
i_{\tau \sigma}: X_{\tau} \rightarrow X_{\sigma}
$$


such that the comorphism

$$
i_{\tau \sigma}^{\#}: k\left[X_{\sigma}\right]=\bigoplus_{\lambda \in \Lambda^{+} \cap \sigma} \text { End } V_{\lambda} \rightarrow \bigoplus_{\lambda \in \Lambda^{+} \cap \tau} \text { End } V_{\lambda}=k\left[X_{\tau}\right]
$$

is the obvious projection.

Clearly, the $i_{\tau \sigma}$ define a directed system of reductive varieties, indexed by the poset $\Sigma / W$. We introduce additional twists of this system, as follows. Consider a collection

$$
t=\left\{t_{\tau \sigma} \in \operatorname{Aut}^{G \times G}\left(X_{\tau}\right) \mid \tau \prec \sigma\right\}
$$

satisfying

$$
t_{\sigma^{\prime \prime} \sigma}=\left.t_{\sigma^{\prime \prime} \sigma^{\prime}} \circ t_{\sigma^{\prime} \sigma}\right|_{X_{\sigma^{\prime \prime}}}
$$

for all triples $\sigma^{\prime \prime} \prec \sigma^{\prime} \prec \sigma$ (note that every automorphism of $X_{\sigma^{\prime}}$ leaves $X_{\sigma^{\prime \prime}}$ invariant). This gives us a twisted directed system of reductive varieties

$$
t_{\tau \sigma} i_{\tau \sigma}: X_{\tau} \rightarrow X_{\sigma}
$$

Definition 5.2. $X_{\Sigma, t}=\lim _{\longrightarrow} X_{\sigma}$.

Note that the twists are just the 1-cocycles of the complex of diagonalizable groups

$$
0 \rightarrow \bigoplus_{\bar{\sigma} \in \Sigma / W} \operatorname{Aut}^{G \times G}\left(X_{\sigma}\right) \rightarrow \bigoplus_{\bar{\tau} \prec \bar{\sigma}} \operatorname{Aut}^{G \times G}\left(X_{\tau}\right) \rightarrow \cdots
$$

with the obvious differential. We denote this complex by $C^{*}(\Sigma / W$, Aut $)$, with cocycle groups $Z^{i}(\Sigma / W$, Aut $)$ and cohomology groups $H^{i}(\Sigma / W$, Aut). As in the toric case (see Ale02 Section 2.3), the following is easy to prove:

Proposition 5.3. $\quad$ (1) $X_{\Sigma, t}$ is a stable reductive variety (for $G$ ). Its irreducible components are the varieties $X_{\sigma}$ where $\sigma \in \Sigma$ is a maximal cone such that $\sigma^{0}$ meets $\Lambda_{\mathbb{R}}^{+}$.

(2) The $G \times G$-orbits in $X_{\Sigma, t}$ are in bijection with the $W$-orbits of cones in $\Sigma$.

(3) $\operatorname{Aut}^{G \times G}\left(X_{\Sigma, t}\right)=H^{0}(\Sigma / W$, Aut $)$.

(4) The set of isomorphism classes of $G \times G$-varieties $X_{\Sigma, t}$ for a fixed $W$-complex $\Sigma$ is $H^{1}(\Sigma / W$, Aut $)$.

Together with the results of Subsection 4.2, this implies the general version of the "toric correspondence":

Theorem 5.4. The stable reductive varieties for $G$ are precisely the varieties $X_{\Sigma, t}$, where $\Sigma$ is a $W$-complex of cones, and $t \in Z^{1}(\Sigma / W$, Aut $)$.

Thus, the assignment $X \mapsto X^{\operatorname{diag} T}$ defines a bijective correspondence from the stable reductive varieties (for $G$ ), to the stable toric varieties (for $T)$ with a compatible $W$-action. This correspondence preserves orbits and automorphism groups.

Moreover, the closed $\operatorname{diag} G$-orbits in $X$ are exactly the orbits in $X^{\operatorname{diag} T}$; the union of these orbits contains a dense subset of every $G \times G$-orbit, and the natural morphism $X^{\operatorname{diag} T} / W \rightarrow X / / \operatorname{diag} G$ is an isomorphism. 
Finally, $X$ is self-adjoint for a unique automorphism fixing pointwise $X^{\operatorname{diag} T}$.

Proof. Let $\left(X_{i}=\overline{\mathcal{O}}_{i}\right)$ be the finite set of $G \times G$-orbit closures in a stable reductive variety $X$. For every $i, k\left[X_{i}\right]$ embeds into $k\left[\mathcal{O}_{i}\right]$. Hence, $X_{i}$ is diagonal and multiplicity-free. The connectedness of stabilizers implies that the lattice $\Lambda_{X_{i}}$ is saturated in $\Lambda$ (by Lemma 3.2), and that the normalization morphism $\nu_{i}: \tilde{X}_{i} \rightarrow X_{i}$ is bijective (by Lemma 2.5). Therefore, the normalizations are reductive varieties $\tilde{X}_{i}=X_{\sigma_{i}}$ and the glueing data $t$ for $X_{i}$ are the same as the glueing data for $\tilde{X}_{i}$. The inclusions between $X_{i}$ 's define on the set $\left\{\sigma_{i}\right\}$ the structure of a complex of cones $\Sigma$. The collection of morphisms $\tilde{X}_{i} \rightarrow X$ defines a finite bijective morphism $\pi: X_{\Sigma, t} \rightarrow X$. Since $X$ is seminormal, $\pi$ is an isomorphism.

\section{Self-adjoint Stable Reductive Semigroups}

We study the relations between stable reductive varieties, self-adjoint stable reductive semigroups, and reductive monoids. We begin with the following observation.

Lemma 6.1. Any normal reductive monoid with unit group a quotient of $G$ by a connected normal subgroup is a self-adjoint reductive semigroup for $G$.

Conversely, any reductive semigroup for $G$ having an identity element is a normal reductive monoid, with unit group a quotient of $G$ by a connected normal subgroup.

Proof. Let $X$ be a normal reductive monoid with unit group $G / G_{1}$, where $G_{1}$ is a connected normal subgroup. Then the $G \times G$-action on $X$ factors through an action of $G / G_{1} \times G / G_{1}$. Thus, we may replace $G$ by $G / G_{1}$, and assume that the unit group is $G$.

By Rit98 Theorem 2, $X$ is affine. And since $G$ is dense in $X$, the algebra $k[X]$ is a normal $G \times G$-invariant subalgebra of $k[G]$. Thus, $X$ is multiplicityfree and diagonal, and $\Lambda_{X}^{+}$is saturated in $\Lambda$. By Proposition 2.4 $X$ satisfies conditions (1) and (2) of Definition 1.3

Let $m: X \times X \rightarrow X$ be the multiplication, with identity element 1 . Clearly, $m$ satisfies the equivariance condition of Definition 1.4. Thus, it factors through a morphism $m / / G:(X \times X) / / G \rightarrow X$, the quotient being for the $G$-action defined by $g \cdot\left(x_{1}, x_{2}\right)=\left((1, g) x_{1},(g, 1) x_{2}\right)$. Note that $m / / G$ is surjective (since $m(x, 1)=x$ ) and birational (since $m^{-1}(1)=\{(x, y) \in$ $G \times G \mid x y=1\}$ is a unique $G$-orbit). Since $X$ is affine and normal, it follows that $m / / G$ is an isomorphism.

Thus, $X$ is a reductive semigroup for $G$. Moreover, the involution $\Theta_{G}$ : $g \mapsto \theta\left(g^{-1}\right)$ extends to an involution $\Theta_{X}$ of $X$, by (the proof of) Proposition 4.5. Clearly, $\Theta_{X}$ makes $X$ self-adjoint.

Conversely, let $X$ be a reductive semigroup for $G$, equipped with an identity element 1 . Then $\left(1 g^{-1}\right)(g 1)=1$ for any $g \in G$, by the equivariance condition. Thus, $g 1$ has a left inverse. By Rit98 Corollary 1, it follows 
that $g 1$ is a unit, with inverse $1 g^{-1}$. As a consequence, $g 1 g^{-1}=1$, so that $g 1=1 g$ for all $g \in G$. This implies that the map $g \mapsto g 1$ is an algebraic group homomorphism from $G$ to the unit group $G(X)$. Moreover, $G \times G$ has only finitely many orbits in $X$ (by Proposition 2.3), and hence in $G(X)$ (where $G \times G$ acts by left and right multiplication). Therefore, the image of $G$ in $G(X)$ is a subgroup of finite index. But $G(X)$ is a connected algebraic group, by Rit98 Theorem 1. So $G(X)$ is the quotient of $G$ by a normal subgroup, which must be connected since all isotropy groups of $G \times G$ are.

Lemma 6.2. Let $X$ be a self-adjoint stable reductive semigroup for $G$. Then $X$ is a multiplicity-free stable reductive variety for $G$. Moreover, any closed $G \times G$-subvariety $Y$ is an ideal (that is, $Y$ contains all products $x y$, yx where $x \in X, y \in Y)$ and is invariant under the automorphism $\Theta_{X}$.

Proof. We have an equivariant isomorphism $k[X] \simeq\left(k[X] \otimes_{k} k[X]\right)^{G}$. It identifies the subalgebra $k[X]^{G \times G}$ to $\left(k[X] \otimes_{k} k[X]\right)^{G \times G \times G}$ (where $G \times G \times G$ acts by $\left(g_{1}, g_{2}, g_{3}\right) \mapsto\left(g_{1}, g_{2}\right) \otimes\left(g_{2}, g_{3}\right)$.) The latter contains the subalgebra $k[X]^{G \times G} \otimes_{k} k[X]^{G \times G}$, so that

$$
k[X]^{G \times G} \otimes_{k} k[X]^{G \times G} \subseteq k[X]^{G \times G}
$$

as a subalgebra. Since $k[X]^{G \times G}$ is a finitely generated $k$-algebra, this is only possible if $k[X]^{G \times G}=k$. Thus, all multiplicities of the $G \times G$-module $k[X]$ are finite.

Write

$$
k[X]=\bigoplus_{\lambda, \mu \in \Lambda} m_{\lambda, \mu} V_{\lambda}^{*} \otimes_{k} V_{\mu}
$$

where the $m_{\lambda, \mu}$ are non-negative integers. Then $m_{\lambda, \mu}=m_{\mu, \lambda}$ (since $X$ is self-adjoint.) Moreover,

$$
m_{\lambda, \mu}=\sum_{\nu \in \Lambda^{+}} m_{\lambda, \nu} m_{\nu, \mu}
$$

since $X$ is isomorphic to $(X \times X) / / G$. It follows that

$$
m_{\lambda, \lambda}=m_{\lambda, \lambda}^{2}+\sum_{\nu \neq \lambda} m_{\lambda, \nu}^{2}
$$

so that $m_{\lambda, \lambda} \leq 1$ and $m_{\lambda, \nu}=$ for any $\nu \neq \lambda$. Thus, $X$ is diagonal and multiplicity-free. By Proposition 2.7 it follows that $X$ is a stable reductive variety.

Write $k[X]=\bigoplus_{\lambda \in \Lambda_{X}^{+}}$End $V_{\lambda}$. By the equivariance condition, the comorphism $m^{\#}$ of the multiplication $m$ sends every End $V_{\lambda}$ to End $V_{\lambda} \otimes_{k}$ End $V_{\lambda}$. Since $Y$ is $G \times G$-invariant, its ideal $I_{Y} \subset k[X]$ is a partial sum of simple submodules End $V_{\lambda}$. It follows that

$$
m^{\#}\left(I_{Y}\right) \subseteq I_{Y} \otimes_{k} I_{Y}=\left(k[X] \otimes_{k} I_{Y}\right) \cap\left(I_{Y} \otimes_{k} k[X]\right) .
$$

In other words, $m\left((X \times Y) \cup(Y \times X) \subseteq Y\right.$. Likewise, since each End $V_{\lambda}$ is invariant under $\Theta_{X}$, the same holds for $I_{Y}$, and for $Y$. 
In particular, any self-adjoint reductive semigroup is a reductive variety. We now establish the converse:

Theorem 6.3. Any reductive variety $X$ admits a structure of self-adjoint reductive semigroup for $G$; then $X^{\operatorname{diag} T}$ is a subsemigroup, which makes it a stable reductive semigroup for $T$. Moreover, $\operatorname{Aut}^{G \times G}(X)$ acts simply transitively on the set of all reductive semigroup structures on $X$.

Proof. Let $X=X_{\sigma}$ be a reductive variety and let $K=K_{\sigma}$. Write

$$
k[X]=\bigoplus_{\lambda \in \Lambda^{+} \cap \sigma} \text { End } V_{\lambda}
$$

and recall that the multiplication of $k[X]$ is defined by

$$
f_{\lambda} \cdot f_{\mu}=\sum_{\nu \in \Lambda^{+} \cap \sigma, \nu \leq_{K} \lambda+\mu} \operatorname{Tr}_{1}\left(p_{\lambda \mu}^{\nu}\left(f_{\lambda} \otimes f_{\mu}\right)\right),
$$

for any $f_{\lambda} \in$ End $V_{\lambda}, f_{\mu} \in$ End $V_{\mu}$. Let $m: X \times X \rightarrow X$ be a morphism satisfying the equivariance condition of Definition 1.4. Then, as noted in the proof of Lemma [6.2, the comorphism $m^{\#}: k[X] \rightarrow k[X] \otimes_{k} k[X]$ sends any End $V_{\lambda}$ to End $V_{\lambda} \otimes_{k}$ End $V_{\lambda}$. Let

$$
m_{\lambda}^{\#}: \text { End } V_{\lambda} \rightarrow \text { End } V_{\lambda} \otimes_{k} \text { End } V_{\lambda}
$$

be the restriction; identify End $V_{\lambda}$ to $V_{\lambda}^{*} \otimes_{k} V_{\lambda}$, and End $V_{\lambda} \otimes_{k}$ End $V_{\lambda}$ to $V_{\lambda}^{*} \otimes_{k} V_{\lambda} \otimes_{k} V_{\lambda}^{*} \otimes_{k} V_{\lambda}$. Then by equivariance, there exists $c_{\lambda} \in k$ such that

$$
m_{\lambda}^{\#}(\eta \otimes v)=c_{\lambda} \sum_{i} \eta \otimes v_{i} \otimes \eta_{i} \otimes v,
$$

where $\left(v_{i}\right)$ is a basis of $V_{\lambda}$ consisting of $T$-eigenvectors, and $\left(\eta_{i}\right)$ is the dual basis. Moreover, $c_{\lambda} \neq 0$ by the invariance condition.

We claim that $m^{\#}: k[X] \rightarrow k[X] \otimes_{k} k[X]$ is compatible with the multiplication if and only if: $c_{\lambda} c_{\mu}=c_{\nu}$ for all $\lambda, \mu, \nu$ in $\Lambda^{+} \cap \sigma$ such that the product End $V_{\lambda}$. End $V_{\mu}$ contains End $V_{\nu}$, that is: $V_{\lambda} \otimes_{k} V_{\mu}$ contains $V_{\nu}$, and $\nu \leq_{K} \lambda+\mu$. To check this, we identify every End $V_{\lambda}$ to its dual space, via the bilinear form $(u, v) \mapsto \operatorname{Tr}(u v)$. This identifies $m_{\lambda}^{\#}$ to the dual of the map

$$
\text { End } V_{\lambda} \otimes_{k} \text { End } V_{\lambda} \rightarrow \text { End } V_{\lambda}, \varphi \otimes \psi \mapsto c_{\lambda} \varphi \circ \psi \text {. }
$$

Now the compatibility of $m^{\#}$ with multiplication is equivalent to the commutativity of the following diagram:

$$
\begin{aligned}
& \text { End } V_{\lambda} \otimes_{k} \text { End } V_{\mu} \stackrel{m_{\lambda}^{\#} \otimes m_{\mu}^{\#}}{\longrightarrow} \text { End } V_{\lambda} \otimes_{k} \text { End } V_{\lambda} \otimes_{k} \text { End } V_{\mu} \otimes_{k} \text { End } V_{\mu} \\
& p_{\lambda \mu}^{\nu} \downarrow \\
& \text { End } V_{\nu} \quad \stackrel{m_{\nu}^{\#}}{\longrightarrow} \quad \text { End } V_{\nu} \otimes_{k} \text { End } V_{\nu} \text {. }
\end{aligned}
$$


This amounts to the commutativity of the dual diagram

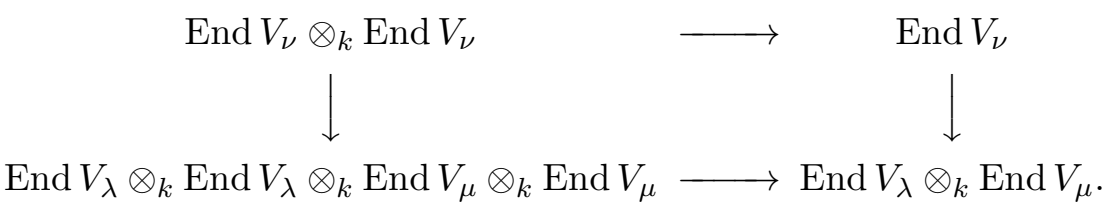

For this, let $\varphi, \psi \in$ End $V_{\nu}$. Then

$$
\left(p_{\lambda \mu}^{\nu}\right)^{*}(\varphi)=1 \otimes \varphi \in \text { End } N_{\lambda \mu}^{\nu} \otimes_{k} \text { End } V_{\nu} \subseteq \text { End } V_{\lambda} \otimes_{k} \text { End } V_{\mu},
$$

so that

$$
c_{\lambda}\left(p_{\lambda \mu}^{\nu}\right)^{*}(\varphi) \circ c_{\mu}\left(p_{\lambda \mu}^{\nu}\right)^{*}(\psi)=c_{\lambda} c_{\mu} 1 \otimes(\varphi \circ \psi)
$$

whereas

$$
c_{\nu}\left(p_{\lambda \mu}^{\nu}\right)^{*}(\varphi \circ \psi)=c_{\nu} 1 \otimes(\varphi \circ \psi) .
$$

This completes the proof of the claim.

Now choose $c_{\lambda}=1$ for any $\lambda \in \Lambda^{+} \cap \sigma$. Then $m^{\#}$ is compatible with multiplication, by that claim. And one checks similarly that $m^{\#}$ is compatible with the automorphism $\Theta_{X}^{\#}$ of $k[X]$, associated with $\Theta_{X}$.

Thus, we obtain a morphism $m: X \times X \rightarrow X$ satisfying the equivariance condition. Since every $m_{\lambda}^{\#}$ defines an isomorphism End $V_{\lambda} \simeq$ (End $V_{\lambda} \otimes_{k}$ End $\left.V_{\lambda}\right)^{G}$, the induced map $(X \times X) / / G \rightarrow X$ is an isomorphism. Moreover, $m$ is associative, as follows from the definition of $m^{\#}$ and associativity of the composition of endomorphisms.

By equivariance, $m: X \times X \rightarrow X$ maps $X^{\operatorname{diag} T} \times X^{\operatorname{diag} T}$ to $X^{\operatorname{diag} T}$. Thus, $X^{\operatorname{diag} T}$ is a subsemigroup of $X$. Clearly, it satisfies the equivariance condition. To check the remaining invariance condition, it suffices to show that the map

$$
m_{\lambda}^{\operatorname{diag} T, \#}: k\left[X^{\operatorname{diag} T}\right]_{\lambda} \rightarrow k\left[X^{\operatorname{diag} T}\right]_{\lambda} \otimes_{k} k\left[X^{\operatorname{diag} T}\right]_{\lambda}
$$

(induced by multiplication) is non-zero for any $\lambda \in W\left(\Lambda^{+} \cap \sigma\right)$, where $k\left[X^{\operatorname{diag} T}\right]_{\lambda}$ denotes the $\lambda$-weight space. By $W$-equivariance, we may assume that $\lambda \in \Lambda^{+} \cap \sigma$. Let $v_{\lambda} \in V_{\lambda}$ be a highest weight vector (of weight $\lambda$ ), and $\eta_{\lambda} \in V_{\lambda}^{*}$ be a lowest weight vector (of weight $-\lambda$ ). Then $\eta_{\lambda} \otimes v_{\lambda} \in$ End $V_{\lambda} \subset k[X]$ restricts to a non-zero function $\varphi_{\lambda} \in k\left[X^{\operatorname{diag} T}\right]_{\lambda}$. Since

$$
m^{\#}\left(\eta_{\lambda} \otimes v_{\lambda}\right)=\sum_{i} \eta_{\lambda} \otimes v_{i} \otimes \eta_{i} \otimes v_{\lambda},
$$

and $v_{\lambda}$ (resp. $\left.\eta_{\lambda}\right)$ is the unique vector of weight $\lambda($ resp. $-\lambda)$ in the basis $\left(v_{i}\right)$ (resp. $\eta_{i}$ ), it follows that

$$
m_{\lambda}^{\operatorname{diag} T, \#}\left(\varphi_{\lambda}\right)=\varphi_{\lambda} \otimes \varphi_{\lambda} .
$$

Finally, note that $\operatorname{Aut}^{G \times G}(X)$ preserves the decomposition of the $G \times$ $G$-module $k[X]$; thus, we may identify $\operatorname{Aut}^{G \times G}(X)$ to the multiplicative group of families $\left(c_{\lambda}\right)_{\lambda \in \Lambda^{+} \cap \sigma}$ of non-zero scalars, satisfying: $c_{\lambda} c_{\mu}=c_{\nu}$ for all $\lambda, \mu, \nu \in \Lambda^{+} \cap \sigma$ such that End $V_{\lambda}$. End $V_{\mu}$ contains End $V_{\nu}$. Together with 
the claim, this proves that any two structures of reductive semigroup on $X$ are conjugate by a unique element of $\operatorname{Aut}^{G \times G}(X)$.

Next we describe the idempotents in a self-adjoint stable reductive semigroup $X$ (that is, the elements $e \in X$ such that $e^{2}=e$ ), generalizing results of Putcha and Renner Put82, Put84, PutRen88 about reductive monoids. Clearly, the subset of idempotents is $\operatorname{diag} G$-invariant and closed in $X$.

Proposition 6.4. Let $X$ be a self-adjoint stable reductive semigroup for $G$. Then the set of idempotents of $X$ meets any $G \times G$-orbit $\mathcal{O}$, along a unique $\operatorname{diag} G$-orbit. Moreover, there exists a unique idempotent $e_{\mathcal{O}}$ in $\mathcal{O}_{0}^{\operatorname{diag} T}$, where $\mathcal{O}_{0}$ denotes the open $B^{-} \times B$-orbit in $\mathcal{O}$.

Proof. By Proposition 4.1 and Lemma 6.2, the closure of $\mathcal{O}$ is a self-adjoint reductive subsemigroup of $X$. Thus, we may assume that $\mathcal{O}$ is open in $X$.

Let $X^{\prime}$ be the closure in $X$ of $\mathcal{O}_{0}^{\operatorname{diag} T}$. By Lemma 6.2 and Theorem 6.3 $X^{\prime}$ is a subsemigroup of $X$, which makes it a reductive semigroup (for $T$ ). Since $X^{\prime}$ is an affine toric variety, it follows easily that every $T \times T$-orbit in $X^{\prime}$ contains a unique idempotent. In particular, $\mathcal{O}_{0}^{\operatorname{diag} T}$ contains a unique idempotent $e=e_{\mathcal{O}}$.

Let $e^{\prime} \in \mathcal{O}$ be another idempotent. Then $e^{\prime}=g e g^{\prime}$ for some $g, g^{\prime} \in G$. Moving $e^{\prime}$ in its $\operatorname{diag} G$-orbit, we may assume that $e^{\prime}=g e$ for some $g \in G$. Then ege $=e$, since $e^{\prime 2}=e^{\prime}$. Let $H=\operatorname{Stab}_{G \times G}(e)$, then the structure of $H$ is given by Proposition 3.1 Since $g_{1} e g_{2}^{-1}=e$ for any $\left(g_{1}, g_{2}\right) \in H$, it follows that $g_{1} e=e=e g_{2}$ for any $g_{1} \in R_{u}\left(P_{I}\right) H_{J}$ and $g_{2} \in R_{u}\left(P_{I}^{-}\right) H_{J}$; moreover, $x e=e x$ for any $x \in L_{K}$. By the Bruhat decomposition, one has $g=g_{2} n x g_{1}$ for some $g_{1} \in R_{u}\left(P_{I}\right), n \in N, x \in L_{K}$ and $g_{2} \in R_{u}\left(P_{I}^{-}\right) H_{J}$. Thus,

$$
e=e g e=e n x e=e\left(n e n^{-1}\right) n x .
$$

As a consequence, $e\left(n e n^{-1}\right) \in \mathcal{O}^{\operatorname{diag} T}$. Since both $e, n e n^{-1}$ are idempotents in the stable toric semigroup $X^{\operatorname{diag} T}$, it follows that $n e n^{-1} \in \mathcal{O}^{\operatorname{diag} T}$. Thus, $n \in L_{I}$ by Proposition [3.4 and since $L_{I}=H_{J} L_{K}$, we may assume that $n=1$. Then $e=e x=x e$, and

$$
e^{\prime}=g e=g_{2} x g_{1} e=g_{2} e=g_{2} e g_{2}^{-1} .
$$

For any idempotent $e$ in a semigroup $X$, the subset

$$
e X e=\{x \in X \mid x=e x=x e\}
$$

is a submonoid, with identity element $e$. If, in addition, $X$ is a self-adjoint stable reductive semigroup, then $e X e$ has a richer structure:

Proposition 6.5. Let e be an idempotent in the self-adjoint stable reductive semigroup $X$. Then the subsets

$$
P=\{g \in G \mid g e \in e G\}, P^{-}=\{g \in G \mid e g \in G e\}
$$


are opposite parabolic subgroups of $G$, with common Levi subgroup

$$
L=\{g \in G \mid g e=e g\} \simeq \operatorname{Stab}_{\operatorname{diag} G}(e) .
$$

Moreover, the subset $e X e$ is a normal reductive submonoid of $X$, with identity element $e$ and unit group the quotient of $\operatorname{Stab}_{\operatorname{diag} G(e)}$ by a connected normal subgroup.

Proof. Note that

$$
e X e \subseteq e X e G \subseteq e X \overline{G e G} \subseteq \overline{G e G},
$$

where the latter inclusion holds by Lemma 6.2 Thus, $e X e=e \overline{G e G} e$, and we may assume that $G e G=\mathcal{O}$ is open in $X$. By Proposition 6.4, we may also assume that $e=e_{\mathcal{O}}$; then the isotropy group $\operatorname{Stab}_{G \times G}(e)$ is described by Proposition 3.1. Since $P$ (resp. $P^{-}$) is the projection of $\operatorname{Stab}_{G \times G}(e)$ to the first (resp. second) copy of $G$, this implies the first assertion.

Clearly, $e X e=\{x \in X \mid x=x e=e x\}$ is closed in $X$, and contains $e G e G e$ as a dense subset. Moreover, $R_{u}(P) L R_{u}\left(P^{-}\right)$is dense in $G$, and $R_{u}(P) e=e R_{u}\left(P^{-}\right)=e$ by Proposition 3.1. Therefore, eLeLe $=e L=L e$ is dense in $e X e$. In fact, the map $g \mapsto g e$ is a homomorphism of $L$ to the unit group $G(e X e)$; hence the image $L e$ is open in that group. But since $e X e$ is irreducible, $G(e X e)$ is connected, so that it equals $L e$. In addition, the isotropy group $\operatorname{Stab}_{L}(e)=\{g \in G \mid g e=e g=e\}$ is connected by Proposition 3.1 and Lemma 3.2 .

It remains to show that the variety $e X e$ is normal. For this, we argue as in the proof of the normality of $\overline{e T e}=X^{\prime}$ (Proposition 4.4). We identify $k[e X e]$ with a $L \times L$-invariant subalgebra of $k\left[L / \operatorname{Stab}_{L}(e)\right]$; the latter identifies in turn with a $L_{K} \times L_{K}$-invariant subalgebra of $k\left[L_{K}\right]$, with the notation of Proposition [3.1] Then the vector space $k[e X e]$ is spanned by the functions on $L_{K} \simeq L_{K} \times L_{K} / \operatorname{diag} L_{K}$ :

$$
(x, y) \mapsto \operatorname{Tr}_{V_{\lambda}}\left(u x p_{\lambda, K} y^{-1}\right)=\operatorname{Tr}_{V_{\lambda}}\left(p_{\lambda, K} y^{-1} u x\right),
$$

that is, by the matrix coefficients of End $V_{\lambda, K}$ (where $\left.\lambda \in \Lambda^{+} \cap \sigma\right)$. It follows that

$$
k[e X e] \simeq \bigoplus_{\lambda \in \Lambda^{+} \cap \sigma} \text { End } V_{\lambda, K}
$$

as a $L_{K} \times L_{K}$-module. This implies normality of $e X e$, for example by using Proposition 2.4 and Lemma 2.5 .

Finally, we characterize self-adjoint stable reductive semigroups among stable reductive varieties.

Proposition 6.6. For a stable reductive variety $X=X_{\Sigma, t}$, the following conditions are equivalent:

(1) $X$ admits a structure of self-adjoint stable reductive semigroup.

(2) The reference map $\rho$ is injective, and the cocycle $t$ is a coboundary.

Then any two structures of stable reductive semigroup are conjugate by a unique automorphism. 
Proof. (1) $\Rightarrow(2)$ Any $\sigma \in \Sigma$ defines a closed irreducible $G \times G$-subvariety $Y_{\sigma} \subseteq X$. By Proposition 6.4 $Y_{\sigma}$ is equipped with a base point $e_{\sigma}$ in its open $B^{-} \times B$-orbit. This defines a unique isomorphism $X_{\sigma} \simeq Y_{\sigma}$ of self-adjoint reductive semigroups, where the semigroup structure on $X_{\sigma}$ is such that the base point is idempotent (there is a unique such structure, by Theorem 6.3 and Proposition 6.4). These isomorphisms identify the inclusions $Y_{\tau} \subseteq Y_{\sigma}$ with the maps $i_{\tau \sigma}: X_{\tau} \rightarrow X_{\sigma}$. Thus, $X$ is isomorphic to the direct limit of the $X_{\sigma}$, with the trivial cocycle $t$. Moreover, $X$ is multiplicity-free by Lemma 6.2] it follows that $\rho$ is injective.

$(2) \Rightarrow(1)$ We may assume that $t$ is constant. Then the preceding argument shows that $X=\lim _{\sigma} X_{\sigma}$ is a self-adjoint semigroup satisfying conditions (1) and (2) of Definition 1.4. Moreover, the multiplication $m: X \times X \rightarrow X$ is surjective (since this holds on every irreducible component). Therefore, the comorphism

$$
m^{\#}: k[X] \rightarrow\left(k[X] \otimes_{k} k[X]\right)^{G}
$$

is injective and $G \times G$-equivariant. On the other hand, the assumptions that $t$ is trivial and $\rho$ is constant imply that $X$ is multiplicity-free; it follows that $m^{\#}$ is an isomorphism.

The final assertion follows from Lemma 6.2 and Theorem 6.3

\section{FAMILIES}

7.1. General remarks. We record some general results on arbitrary families of affine $G$-varieties, in the following sense.

Definition 7.1. A family of affine (irreducible) G-varieties over a scheme $S$ is a scheme $\mathcal{X}$ equipped with a morphism $\pi: \mathcal{X} \rightarrow S$ and with an action of the constant group scheme $G \times S$ over $S$, satisfying the following conditions:

(1) $\pi$ is flat and affine.

(2) Every geometric fiber of $\pi$ is reduced (and irreducible).

This yields a rational action of $G \times S$ on the sheaf $\pi_{*} \mathcal{O}_{\mathcal{X}}$, by Mum94 p. 25. As for families of stable reductive varieties, we will often drop the adjective "affine" and deal with families of $G$-varieties.

Lemma 7.2. Let $\pi: \mathcal{X} \rightarrow S$ be a family of multiplicity-finite (multiplicitybounded, multiplicity-free) $G$-varieties. Then the map $\mathcal{O}_{S} \rightarrow\left(\pi_{*} \mathcal{O}_{\mathcal{X}}\right)^{G}$ is an isomorphism, so that $\pi$ is the categorical quotient by $G$. Moreover, one has an isomorphism of $\mathcal{O}_{S}-G$-modules:

$$
\pi_{*} \mathcal{O}_{\mathcal{X}} \simeq \bigoplus_{\lambda \in \Lambda^{+}} F_{\lambda} \otimes_{k} V_{\lambda}
$$

where all the $F_{\lambda} \simeq\left(\pi_{*} \mathcal{O}_{\mathcal{X}}\right)_{\lambda}^{U}$ are locally free $\mathcal{O}_{S}$-modules of finite rank (of bounded rank, of rank at most 1 ).

As a consequence, $\pi$ factors through a family $\pi / / U: \mathcal{X} / / U \rightarrow S$ of multiplicity-finite (multiplicity-bounded, multiplicity-free) T-varieties. 
Proof. We may assume that $S$ is affine; hence so is $\mathcal{X}$. Let $\mathcal{R}=\Gamma\left(\mathcal{X}, \mathcal{O}_{\mathcal{X}}\right)$ and $\mathcal{R}_{0}=\Gamma\left(S, \mathcal{O}_{S}\right)$. The $\mathcal{R}_{0}$-module $\mathcal{R}$ is flat, so that its direct factor $\mathcal{R}^{G}$ is flat as well. Since every geometric fiber $\mathcal{X}_{\bar{s}}$ is reduced and multiplicity-finite, one has

$$
\mathcal{R}^{G} \otimes_{\mathcal{R}_{0}} k(\bar{s}) \simeq \Gamma\left(\mathcal{X}_{\bar{s}}, \mathcal{O}_{\mathcal{X}_{\bar{s}}}\right)^{G}=\Gamma\left(\mathcal{X}_{\bar{s}}, \mathcal{O}_{\mathcal{X}_{\bar{s}}}\right)^{G(k(\bar{s}))}=k(\bar{s}) .
$$

It follows that the map $\mathcal{R}_{0} \rightarrow \mathcal{R}^{G}$ is an isomorphism. And since $G$ acts rationally on $\mathcal{R}$, one has an isomorphism of $\mathcal{R}_{0}$ - $G$-modules:

$$
\bigoplus_{\lambda \in \Lambda^{+}} \operatorname{Hom}^{G}\left(V_{\lambda}, \mathcal{R}\right) \otimes_{k} V_{\lambda} \rightarrow \mathcal{R}
$$

given by evaluation. Since $\mathcal{R}$ is a flat module over $\mathcal{R}_{0}$, the same holds for each $\operatorname{Hom}^{G}\left(V_{\lambda}, \mathcal{R}\right)$. But this module of covariants is finitely generated over $\mathcal{R}_{0}=\mathcal{R}^{G}$ (since $\mathcal{R}$ is Noetherian), and hence locally free. Moreover, $\operatorname{Hom}^{G}\left(V_{\lambda}, \mathcal{R}\right) \simeq \mathcal{R}_{\lambda}^{U}$. The assertion on ranks follows from the isomorphism

$$
\mathcal{R}_{\lambda}^{U} \otimes_{\mathcal{R}_{0}} k(\bar{s}) \simeq \Gamma\left(\mathcal{X}_{\bar{s}}, \mathcal{O}_{\mathcal{X}_{\bar{s}}}\right)_{\lambda}^{U}=\Gamma\left(\mathcal{X}_{\bar{s}}, \mathcal{O}_{\mathcal{X}_{\bar{s}}}\right)_{\lambda}^{U(k(\bar{s}))}
$$

Together with Proposition 2.4 this implies at once the following

Corollary 7.3. Let $\pi: \mathcal{X} \rightarrow S$ be a family of $G$-varieties, where $S$ is connected. Then the multiplicities of the $G(k(\bar{s}))$-module $\Gamma\left(\mathcal{X}_{\bar{s}}, \mathcal{O}_{\mathcal{X}_{\bar{s}}}\right)$ are independent of the geometric point $\bar{s}$ of $S$. In particular, if some geometric fiber is multiplicity-finite (multiplicity-bounded, multiplicity-free), then so are all geometric fibers.

If, in addition, some geometric fiber is multiplicity-free with a saturated weight set, then all geometric fibers are connected and seminormal.

This defines the Hilbert function $h: \Lambda^{+} \rightarrow \mathbb{N}, \lambda \mapsto \operatorname{rank}\left(F_{\lambda}\right)$ of a family of multiplicity-finite $G$-varieties over a connected scheme $S$.

Another useful observation is the following variant of a rigidity lemma due to Knop (Kno94 Lemma 6.1.)

Lemma 7.4. Let $\pi: \mathcal{X} \rightarrow S$ be a family of irreducible multiplicity-free $G$-varieties. Then the family $\pi / / U: \mathcal{X} / / U \rightarrow S$ is locally trivial.

Proof. Choose $s \in S$. Replacing $S$ by a neighborhood of $s$, we may assume that $S$, and hence $\mathcal{X}$, is affine; replacing $\mathcal{X}$ by $\mathcal{X} / / U$, we may also assume that $G=T$ is a torus. Then

$$
\mathcal{R}=\Gamma\left(\mathcal{X}, \mathcal{O}_{\mathcal{X}}\right)=\bigoplus_{\lambda \in \Lambda} \mathcal{R}_{\lambda}
$$

where each non-zero weight space $\mathcal{R}_{\lambda}$ is a locally free module of rank 1 over $\mathcal{R}_{0}=\Gamma\left(S, \mathcal{O}_{S}\right)$. Moreover, $\mathcal{R}_{\lambda} \neq 0$ if and only if $\lambda \in \Lambda_{X}^{+}$, where $X=\mathcal{X}_{\bar{s}}$. Replacing $T$ by a quotient, we may assume that its character group $\Lambda$ is generated by $\Lambda_{X}^{+}$; then $T(k(\bar{s}))$ has an open orbit $\mathcal{O}$ in $X$, isomorphic to $T(k(\bar{s}))$. 
For any $\lambda, \mu$ in $\Lambda_{X}^{+}$, the multiplication map

$$
\mathcal{R}_{\lambda} \otimes_{\mathcal{R}_{0}} \mathcal{R}_{\mu} \rightarrow \mathcal{R}_{\lambda+\mu}
$$

induces an isomorphism on all geometric fibers; thus, this map is an isomorphism. It follows that any local generator of the $\mathcal{R}_{0}$-module $\mathcal{R}_{\lambda}$ is a non-zero divisor in $\mathcal{R}$.

Choose $\lambda_{0} \in \Lambda_{X}^{+}$in the interior of the cone $C_{X}$, and let $f \in \mathcal{R}_{\lambda_{0}}$ such that $\left.f\right|_{X} \neq 0$. Then $f$ generates $\mathcal{R}_{\lambda_{0}}$ at $s$, hence is a non-zero divisor in $\mathcal{R}$, and the open affine subset $\mathcal{X}_{f}$ intersects $X$ along $\mathcal{O}$. As a consequence,

$$
\mathcal{R}\left[f^{-1}\right]=\bigoplus_{\lambda \in \Lambda} \mathcal{R}\left[f^{-1}\right]_{\lambda}
$$

where every $\mathcal{R}\left[f^{-1}\right]_{\lambda}$ is a locally free $\mathcal{R}_{0}$-module of rank 1 . Choose a basis $\left(\lambda_{1}, \ldots, \lambda_{r}\right)$ of the lattice $\Lambda$; for $1 \leq i \leq r$, let $f_{i} \in \mathcal{R}\left[f^{-1}\right]_{\lambda_{i}}$ such that $\left.f_{i}\right|_{X} \neq 0$. Then, for any $\lambda=n_{1} \lambda_{1}+\cdots+n_{r} \lambda_{r} \in \Lambda$, the Laurent monomial $f_{\lambda}=f_{1}^{n_{1}} \cdots f_{r}^{n_{r}}$ is a generator of the $\mathcal{R}_{0}$-module $\mathcal{R}\left[f^{-1}\right]_{\lambda}$ at $s$. Thus, by shrinking $S$, we may assume that $\mathcal{R}\left[f^{-1}\right]$ is just the ring of Laurent polynomials $\mathcal{R}_{0}\left[f_{1}, f_{1}^{-1}, \ldots, f_{r}, f_{r}^{-1}\right]=\bigoplus_{\lambda \in \Lambda} \mathcal{R}_{0} f_{\lambda}$.

Moreover, regarding $\mathcal{R}$ as a $T$-invariant subalgebra of $\mathcal{R}\left[f^{-1}\right]$, we have

$$
\mathcal{R}_{\lambda}=\mathcal{R}\left[f^{-1}\right]_{\lambda}
$$

for all $\lambda \in \Lambda_{X}^{+}$. Indeed, $\mathcal{R}\left[f^{-1}\right]_{\lambda}$ is the union of the spaces $\mathcal{R}_{\lambda+n \lambda_{0}} f^{-n}$ over all non-negative integers $n$, and the equalities $\mathcal{R}_{\lambda+n \lambda_{0}}=\mathcal{R}_{\lambda} \mathcal{R}_{n \lambda_{0}}=\mathcal{R}_{\lambda} f^{n}$ yield $\mathcal{R}_{\lambda+n \lambda_{0}} f^{-n}=\mathcal{R}_{\lambda}$.

So we have constructed generators $f_{\lambda}$ of the $\mathcal{R}_{\lambda}$ 's, satisfying $f_{\lambda} f_{\mu}=f_{\lambda+\mu}$. This trivializes $\pi$ in a neighborhood of $s$.

Finally, we show how to construct families of $G$-varieties with a prescribed fiber, starting from certain families of $G \times G$-varieties; this will be developed in Subsection 7.5 .

Lemma 7.5. Let $X$ be a $G$-variety and let $\pi: \mathcal{X} \rightarrow S$ be a family of $G \times G$ varieties over a connected scheme, such that $\mathcal{X}_{s_{0}} \simeq G$ for some $s_{0} \in S$ with $k\left(s_{0}\right)=k$. Then the categorical quotient

$$
X * \mathcal{X}=(X \times \mathcal{X}) / / G
$$

(for the action by $g \cdot(x, \xi)=(g x,(g, 1) \xi))$, equipped with the $G$-action induced by the $\{1\} \times G$-action on $\mathcal{X}$, and with the map

$$
\pi_{X}:(X \times \mathcal{X}) / / G \rightarrow \mathcal{X} / / G \rightarrow S,
$$

is a family of $G$-varieties, with fiber at $s_{0}$ isomorphic to $X$.

If, in addition, $X$ is multiplicity-finite (multiplicity-bounded, multiplicityfree), then so are all geometric fibers of $\pi_{X}$. 
Proof. We may assume again that $S$, and hence $\mathcal{X}$, is affine. Let again $\mathcal{R}=\Gamma\left(\mathcal{X}, \mathcal{O}_{\mathcal{X}}\right)$ and $\mathcal{R}_{0}=\Gamma\left(S, \mathcal{O}_{S}\right)$. Then

$$
\mathcal{R}=\bigoplus_{\lambda \in \Lambda^{+}} F_{\lambda} \otimes_{k} V_{\lambda}^{*} \otimes_{k} V_{\lambda}
$$

as a $\mathcal{R}_{0}-G \times G$-module, where every $F_{\lambda}$ is an invertible $\mathcal{R}_{0}$-module (this follows from Lemma 7.2 together with the decomposition

$$
k[G] \simeq \bigoplus_{\lambda \in \Lambda^{+}} V_{\lambda}^{*} \otimes_{k} V_{\lambda}
$$

as a $G \times G$-module.) We also have

$$
k[X]=\bigoplus_{\lambda \in \Lambda^{+}} \operatorname{Hom}^{G}\left(V_{\lambda}, k[X]\right) \otimes_{k} V_{\lambda}
$$

as a $G$-module. It follows that

$$
\Gamma\left(X * \mathcal{X}, \mathcal{O}_{X * \mathcal{X}}\right)=\left(k[X] \otimes_{k} R\right)^{G}=\bigoplus_{\lambda \in \Lambda^{+}} \operatorname{Hom}^{G}\left(V_{\lambda}, k[X]\right) \otimes_{k} F_{\lambda} \otimes_{k} V_{\lambda}
$$

as a $\mathcal{R}_{0}-G$-module, where $\mathcal{R}_{0}$ acts on $F_{\lambda}$, and $G$ acts on $V_{\lambda}$. Thus, $\pi_{X}$ is flat. Moreover, $(X * \mathcal{X})_{\bar{s}} \simeq\left(X \times \mathcal{X}_{\bar{s}}\right) / / G(k(\bar{s}))$ for any geometric point $\bar{s}$ of $S$, so that the geometric fibers of $\pi_{X}$ are reduced. Finally, if $\mathcal{X}_{s_{0}}$ is isomorphic to $G$, then $(X * \mathcal{X})_{s_{0}} \simeq(X \times G) / / G \simeq X$. This proves the first assertion.

If, in addition, $X$ is multiplicity-finite (multiplicity-bounded, multiplicityfree), then the dimensions of all spaces $\operatorname{Hom}^{G}\left(V_{\lambda}, k[X]\right)$ are finite (bounded, at most 1). This implies the second assertion.

7.2. Moduli of embedded stable reductive varieties. Next we show that the families of multiplicity-finite $G$-subvarieties of a fixed $G$-module $V$, with a fixed Hilbert function $h$, admit a fine moduli space. In the case where $G$ is abelian, $G$-modules are nothing but $k$-vector spaces endowed with a grading by the character group of $G$. In that case, existence of the moduli space follows from the construction by Haiman and Sturmfels HS02 of the multigraded Hilbert scheme, parametrizing all homogeneous quotient rings of a graded polynomial ring with a fixed Hilbert function.

As a generalization of this setting to arbitrary $G$, consider a function $h: \Lambda^{+} \rightarrow \mathbb{N}$ and a finite-dimensional $G$-module $V$. Define a functor

$$
\mathcal{M}_{h, V}:(\text { Schemes })^{o} \rightarrow(\text { Sets })
$$

by assigning to $S$ the set of all multiplicity-finite subfamilies $\pi: \mathcal{X} \rightarrow S$ of $(V \times S \rightarrow S)$, with Hilbert function $h$.

Theorem 7.6. The functor $\mathcal{M}_{h, V}$ is representable by a scheme $M_{h, V}$ which is quasiprojective over $k$.

Proof. Note that we may define families of affine $G$-schemes by dropping condition (2) (on reducedness of geometric fibers) in Definition [7.1 the 
notions of multiplicity-finiteness and Hilbert function adapt easily to such families. Thus, we may consider the functor

$$
\mathcal{H}_{h, V}:(\text { Schemes })^{o} \rightarrow(\text { Sets })
$$

by assigning to $S$ the set of all closed $G$-invariant subschemes $\mathcal{X} \subseteq V \times S$, flat and multiplicity-finite over $S$ with Hilbert function $h$. We will prove that $\mathcal{H}_{h, V}$ is representable by a quasi-projective scheme $H_{h, V}$, and then that $\mathcal{M}_{h, V}$ is representable by an open subscheme of $H_{h, V}$.

For this, we reduce to a situation where the main result of Haiman and Sturmfels applies, as follows. Choose a regular dominant one-parameter subgroup $\gamma: \mathbb{G}_{m} \rightarrow T$. Then the evaluation map

$$
\Lambda \rightarrow \mathbb{Z}, \lambda \mapsto\langle\lambda, \gamma\rangle
$$

satisfies the following properties:

(1) $\langle\lambda, \gamma\rangle \geq 0$ for all $\lambda \in \Lambda^{+}$.

(2) $\langle\nu, \gamma\rangle \leq\langle\lambda, \gamma\rangle+\langle\mu, \gamma\rangle$ for all $\lambda, \mu, \nu$ in $\Lambda^{+}$such that the $G$-module $V_{\nu}$ occurs in the decomposition of $V_{\lambda} \otimes_{k} V_{\mu}$.

(3) For any non-negative integer $n$ and any character $\chi$ of the center $Z(G)$, the set

$$
\left\{\lambda \in \Lambda^{+} \mid\langle\lambda, \gamma\rangle=n \text { and }\left.\lambda\right|_{Z(G)}=\chi\right\}
$$

is finite.

Now consider an affine scheme $X$ with a $G$-action, and the corresponding $G$-algebra

$$
R=k[X]=\bigoplus_{\lambda \in \Lambda^{+}} \operatorname{Hom}^{G}\left(V_{\lambda}, R\right) \otimes_{k} V_{\lambda}
$$

For any non-negative integer $n$, let

$$
R_{\leq n}=\bigoplus_{\lambda \in \Lambda^{+},\langle\lambda, \gamma\rangle \leq n} \operatorname{Hom}^{G}\left(V_{\lambda}, R\right) \otimes_{k} V_{\lambda} .
$$

This yields an ascending filtration of $R$ by $G$-submodules, and (2) shows that it is an algebra filtration. Let $z$ be a variable, and let

$$
\mathcal{R}=\bigoplus_{n \geq 0} R_{\leq n} z^{n}
$$

be the corresponding Rees algebra. This is a graded $G$-invariant subalgebra of $R[z]$, containing $k[z]$. Any $G$-invariant ideal $I \subseteq R$ yields a graded $G$ invariant ideal

$$
\mathcal{I}=\bigoplus_{n \geq 0}\left(I \cap R_{\leq n}\right) z^{n} \subseteq \mathcal{R},
$$

and hence a graded $G$-algebra $\overline{\mathcal{R}}=\mathcal{R} / \mathcal{I}$. One checks easily that the assignment $I \mapsto \mathcal{I}$ sets up a bijective correspondence from $G$-invariant ideals of $R$, to those graded $G$-invariant ideals $\mathcal{I}$ of $\mathcal{R}$ such that $z$ is a non-zero divisor in $\mathcal{R} / \mathcal{I}$. Moreover, $I$ is prime (radical) if and only if so is $\mathcal{I}$. Finally, by (3), 
the $G$-algebra $R / I$ is multiplicity-finite if and only if so is the $\mathbb{G}_{m} \times Z(G)$ algebra $\mathcal{R} / \mathcal{I}$, where the action of $\mathbb{G}_{m}$ is defined by the compatible gradings of $\mathcal{R}$ and $\mathcal{I}$.

Another graded $G$-invariant ideal of $\mathcal{R}$ is

$$
\mathcal{J}=\mathcal{I}+z \mathcal{R}=\bigoplus_{n=0}^{\infty}\left(R_{\leq n-1}+I \cap R_{\leq n}\right) z^{n} .
$$

Note the isomorphisms of $G$-modules

$$
\mathcal{R}_{n} / \mathcal{J}_{n} \simeq R_{=n} / I_{=n} \simeq \overline{\mathcal{R}}_{\leq n} / \overline{\mathcal{R}}_{\leq n-1}
$$

with evident notation. Thus, the Hilbert functions of $R / I$ (as a $G$-algebra), of $\mathcal{R} / \mathcal{I}$ and of $\mathcal{R} / \mathcal{J}$ (as $\mathbb{G}_{m} \times G$-algebras) determine each other. In particular, the Hilbert function of $R / I$ as a $G$-algebra determines that of $\mathcal{R} / \mathcal{I}$ and $\mathcal{R} / \mathcal{J}$ as $\mathbb{G}_{m} \times Z(G)$-algebras.

Next let $\mathcal{X} \subseteq V \times S$ be a $G$-invariant closed subscheme. Let

$$
R=\operatorname{Sym}^{*}\left(V^{*}\right) \otimes_{k} \mathcal{O}_{S}
$$

be the direct image of the structure sheaf $\mathcal{O}_{V \times S}$ under projection to $S$. Then $\mathcal{X}$ yields a $G$-invariant sheaf of ideals $I \subseteq R$, and hence a $\mathbb{G}_{m} \times G$-invariant sheaf of ideals $\mathcal{I}$ in the Rees algebra

$$
\mathcal{R}=\left(\bigoplus_{n=0}^{\infty} \operatorname{Sym}^{*}\left(V^{*}\right)_{\leq n} z^{n}\right) \otimes_{k} \mathcal{O}_{S} .
$$

The argument of Lemma 7.2 shows that $\mathcal{X}$ is flat over $S$ if and only if so is $\mathcal{R} / \mathcal{I}$. Then $\mathcal{J}=\mathcal{I}+z \mathcal{R}$ is another $\mathbb{G}_{m} \times G$-invariant sheaf of ideals, and $\mathcal{R} / \mathcal{J}$ is flat over $S$, too.

By HS02 Theorem 1.1, the families of pairs $(\mathcal{R} / \mathcal{I}, \mathcal{R} / \mathcal{J})$ of $\mathbb{G}_{m} \times Z(G)$ invariant quotients of $\mathcal{R}$ with fixed Hilbert functions (determined by $h$ ) are parametrized by a quasi-projective scheme $S_{h, V}$. The $\mathcal{O}_{S_{h, V}}$-modules $\mathcal{R} / \mathcal{I}$ and $\mathcal{R} / \mathcal{J}$ split up into direct sums of locally free modules of finite rank according to the action of $\mathbb{G}_{m} \times Z(G)$. By Lemmas 7.7 and 7.8 below, those pairs where $\mathcal{I}+z \mathcal{R}=\mathcal{J}$ and both $\mathcal{I}, \mathcal{J}$ are $G$-invariant are parametrized by a closed subscheme $H_{h, V}$ of $S_{h, V}$.

We claim that $H_{h, V}$ represents the functor $\mathcal{H}_{h, V}$. By the preceding discussion, it suffices to check that $z$ is a non-zero divisor in $\mathcal{R} / \mathcal{I}$. Equivalently, the multiplication by $z$ fits into exact sequences

$$
0 \rightarrow \mathcal{R}_{n-1} / \mathcal{I}_{n-1} \rightarrow \mathcal{R}_{n} / \mathcal{I}_{n} \rightarrow \mathcal{R}_{n} / \mathcal{J}_{n} \rightarrow 0
$$

for all $n$. But these sequences are right exact and split up (under the $\mathbb{G}_{m}$-action) into direct sums of sequences involving only locally free $\mathcal{O}_{H_{h, V}}$ modules of finite rank. Hence their exactness is equivalent to a collection of numerical conditions on these ranks, i.e., it only depends on the Hilbert function. This proves our claim.

It remains to show that $\mathcal{M}_{h, V}$ is representable by an open subscheme of $H_{h, V}$. For this, we consider the universal family $\pi: U_{h, V} \rightarrow H_{h, V}$. We are 
interested in the locus of $H_{h, V}$ where the fibers of $\pi$ are reduced, and we know that the preimage of this locus in $U_{h, V}$ is open and invariant under the natural $G$-action. To conclude, note that $\pi$ maps any closed $G$-invariant subscheme to a closed subscheme, since it factors as the quotient $U_{h, V} \rightarrow$ $U_{h, V} / / G$ followed by a finite morphism $U_{h, V} / / G \rightarrow H_{h, V}$.

Lemma 7.7. Let $S$ be a scheme, $V$ a k-vector space, and let $F_{1}, F_{2}$ be two subsheaves of $V \otimes_{k} \mathcal{O}_{S}$ such that the quotients $V \otimes_{k} \mathcal{O}_{S} / F_{1}=Q_{1}$, $V \otimes_{k} \mathcal{O}_{S} / F_{2}=Q_{2}$ are locally free of finite rank. Then there exists a closed subscheme $S\left(F_{1}, F_{2}\right) \subseteq S$ satisfying the following universal property: for any morphism $f: S^{\prime} \rightarrow S, f^{*} F_{1}$ is a subsheaf of $f^{*} F_{2}$ if and only if $f$ factors through $S\left(F_{1}, F_{2}\right)$.

Proof. By shrinking, we can assume $S$ to be affine, $S=\operatorname{Spec} R$, and $Q_{1}, Q_{2}$ to be freely generated by the images of certain vectors of $V$. Then each $F_{i}$ contains $V_{i} \otimes_{k} \mathcal{O}_{S}$ for some subspace $V_{i} \subseteq V$ of finite codimension. Replacing $V$ with $V / V_{1} \cap V_{2}$, we can assume that $V$ is finite dimensional. Shrinking again, we can also assume that both $F_{1}, F_{2}$ are free with bases $\left\{a_{1}, \ldots, a_{n_{1}}\right\},\left\{b_{1}, \ldots, b_{n_{2}}\right\}$ respectively. The second basis can be extended to a basis $\left\{b_{1}, \ldots, b_{n}\right\}$ of $V \otimes_{k} \mathcal{O}_{S}$. Write the basis of $F_{1}$ as an $n \times n_{1}$ matrix $\left(A_{i j}\right)$ with entries in $R$. Then $S\left(F_{1}, F_{2}\right)$ is the zero scheme of entries $A_{i j}$ with $i>n_{2}$.

Lemma 7.8. Let $S$ be a scheme, $V$ a rational $G$-module, and $F \subseteq V \otimes_{k} \mathcal{O}_{S}$ a subsheaf such that the quotient $V \otimes_{k} \mathcal{O}_{S} / F$ is locally free of finite rank. Then there exists a closed subscheme $S_{F} \subseteq S$ satisfying the following universal property: for any morphism $f: S^{\prime} \rightarrow S, f^{*} F$ is a G-invariant subsheaf of $V \otimes_{k} \mathcal{O}_{S^{\prime}}$ if and only if $f$ factors through $S_{F}$.

Proof. Indeed, $S_{F}$ is the intersection of the closed subschemes $S\left(F, g^{*} F\right)$ for $g \in G$, which exist by the previous Lemma.

7.3. Local isotriviality. An important consequence of the classification of reductive varieties (Theorem 4.8) is the following

Corollary 7.9. Let $\pi: \mathcal{X} \rightarrow S$ be a family of reductive varieties over an integral scheme. Then there exists an étale morphism $\varphi: S^{\prime} \rightarrow S$ such that the pull-back family $\mathcal{X} \times{ }_{S} S^{\prime} \rightarrow S^{\prime}$ is trivial. In particular, $\pi$ admits a general fiber.

Proof. We may assume that $S$, and hence $\mathcal{X}$, are affine. Let $\mathcal{R}=\Gamma\left(\mathcal{X}, \mathcal{O}_{\mathcal{X}}\right)$ and $\mathcal{R}_{0}=\Gamma\left(S, \mathcal{O}_{S}\right)$. Write

$$
\mathcal{R}=\bigoplus_{\lambda \in \Lambda^{+}} F_{\lambda} \otimes_{k} \text { End } V_{\lambda}
$$

as a $\mathcal{R}_{0^{-}} G \times G$-module; then $\mathcal{R}_{0}=F_{0}$. By shrinking $S$, we may further assume that any non-zero $F_{\lambda}$ is generated by some $f_{\lambda}$, satisfying $f_{\lambda} \cdot f_{\mu}=$ $f_{\lambda+\mu}$ (Lemma 7.4). 
On the other hand, the geometric generic fiber of $\pi$ is a reductive variety over $k(\bar{S})$; let $C, K$ be the corresponding combinatorial data. Then

$$
k(\bar{S}) \otimes_{\mathcal{R}_{0}} \mathcal{R}=\bigoplus_{\lambda \in \Lambda \cap C} k(\bar{S}) \otimes_{k} \text { End } V_{\lambda}=k(\bar{S}) \otimes_{k} k\left[X_{C, K}\right]
$$

as a $k(\bar{S})$-algebra. It follows that each $F_{\lambda}$ is contained in $k(\bar{S})$, and is nonzero if and only if $\lambda \in \Lambda \cap C$. This yields elements $f_{\lambda} \in k(\bar{S})^{*}$, which are contained in some finite extension $L$ of $k(S)$ by the first step of the proof. So we obtain an algebra isomorphism

$$
L \otimes_{\mathcal{R}_{0}} \mathcal{R}=L \otimes_{k} k\left[X_{C, K}\right]
$$

which implies our statement.

7.4. One-parameter degenerations. We construct certain families of stable reductive varieties over the affine line; these will provide local models for all one-parameter families of stable reductive varieties. We begin with families whose generic fiber is geometrically irreducible.

Definition 7.10. A $W$-admissible height function is a map $h: \sigma \rightarrow \mathbb{R}$, where $\sigma$ is a $W$-admissible cone in $\Lambda_{\mathbb{R}}$, satisfying the following conditions:

(1) (on rationality) $h(\lambda) \in \mathbb{Q}$ for any $\lambda \in \sigma \cap \Lambda$.

(2) (on invariance) $h(w x)=h(x)$ for any $w \in \operatorname{Stab}_{W}(\sigma)$ and $x \in \sigma$.

(3) (on convexity) $h\left(x_{1}+x_{2}\right) \leq h\left(x_{1}\right)+h\left(x_{2}\right)$ for all $x_{1}, x_{2}$ in $\sigma$.

(4) (on piecewise linearity) $h$ is linear on each cone of some finite subdivision of $\sigma$.

In particular, $h$ is positively homogeneous.

We then denote by $\Sigma_{h}$ the set of cones in $\Lambda_{\mathbb{R}}$ consisting of all $W$-translates of the maximal cones where $h$ is linear, together with their faces. Then the union $\left|\Sigma_{h}\right|$ of these cones is just $\bigcup_{w \in W} w \sigma$. Clearly, $\Sigma_{h}$ is a $W$-complex of cones, the reference map $\left|\Sigma_{h}\right| \rightarrow \Lambda_{\mathbb{R}}$ being the inclusion.

Definition 7.11. Let $X_{h}$ be the stable reductive variety associated with the $W$-complex of cones $\Sigma_{h}$ and with the trivial cocycle $t$.

Note that $X_{h}$ is unchanged when replacing $h$ by a positive rational multiple.

We now construct a one-parameter family of stable reductive varieties with general fiber $X_{\sigma}$ and special fiber $X_{h}$. Let $\tilde{G}=G \times \mathbb{G}_{m}$; this is a connected reductive group with maximal torus $\tilde{T}=T \times \mathbb{G}_{m}$, weight lattice $\tilde{\Lambda}=\Lambda \times \mathbb{Z}$, and Weyl group isomorphic to $W$. Let

$$
\tilde{\sigma}_{h}=\left\{(x, y) \in \tilde{\Lambda}_{\mathbb{R}}=\Lambda_{\mathbb{R}} \times \mathbb{R} \mid x \in \sigma, y \in \mathbb{R}, h(x) \leq y\right\} .
$$

One easily checks that $\tilde{\sigma}_{h}$ is a $W$-admissible cone in $\tilde{\Lambda}_{\mathbb{R}}$; let $\tilde{X}_{h}$ be the corresponding reductive variety (for $\tilde{G}$ ). Since $\tilde{\sigma}_{h}$ contains $\{0\} \times \mathbb{R}_{\geq 0}$, we obtain a non-zero map

$$
\pi_{h}: \tilde{X}_{h} \rightarrow \mathbb{A}^{1}
$$


which is $G \times G$-invariant and $\mathbb{G}_{m} \times \mathbb{G}_{m}$-equivariant (where $\mathbb{G}_{m} \times \mathbb{G}_{m}$ acts on $\mathbb{A}^{1}$ by $\left.\left(t_{1}, t_{2}\right) z=t_{1} t_{2}^{-1} z\right)$.

Proposition 7.12. $\pi_{h}$ is surjective, and $\pi_{h}^{-1}\left(\mathbb{A}^{1}-\{0\}\right) \simeq X_{\sigma} \times\left(\mathbb{A}^{1}-\{0\}\right)$ as $\tilde{G} \times \tilde{G}$-varieties.

Moreover, the fiber of $\pi_{h}$ at 0 is reduced if and only if: $h(\lambda) \in \mathbb{Z}$ for any $\lambda \in \Lambda \cap \sigma$. Then $\pi_{h}$ is a flat family of stable reductive varieties, and its fiber at 0 is isomorphic to $X_{h}$.

Proof. Let $K=K_{\sigma}$ and $\mathcal{X}=\tilde{X}_{h}$. Write

$$
k[\tilde{G}]_{(K)}=k[G]_{(K)}\left[z, z^{-1}\right] \simeq \bigoplus_{\lambda \in \Lambda^{+}, n \in \mathbb{Z}} z^{n} \text { End } V_{\lambda},
$$

where $z$ is the coordinate function on $\mathbb{G}_{m}$. Then

$$
k[\mathcal{X}] \simeq \bigoplus_{\lambda \in \Lambda^{+} \cap \sigma, n \in \mathbb{Z}, h(\lambda) \leq n} z^{n} \text { End } V_{\lambda}
$$

as a subalgebra of $k[G]_{(K)}\left[z, z^{-1}\right]$, and the map $\pi_{h}: \mathcal{X} \rightarrow \mathbb{A}^{1}$ corresponds to the inclusion $k[z] \subseteq k[\mathcal{X}]$. Moreover, one has $k[\mathcal{X}]\left[z^{-1}\right]=k\left[X_{\sigma}\right]\left[z, z^{-1}\right]$. This trivializes $\pi_{h}$ over $\mathbb{A}^{1}-\{0\}$.

For any $n \in \mathbb{Z}$, let

$$
k\left[X_{\sigma}\right]_{\leq n}=\bigoplus_{\lambda \in \Lambda^{+} \cap \sigma, h(\lambda) \leq n} \text { End } V_{\lambda}
$$

Then the $k\left[X_{\sigma}\right]_{\leq n}$ define an ascending filtration of the algebra $k\left[X_{\sigma}\right]$ (indeed, if $\lambda, \mu, \nu$ are in $\Lambda^{+} \cap \sigma$ and satisfy $\nu \leq_{K} \lambda+\mu$, then $\nu \in \operatorname{Conv}\left(W_{K} \lambda\right)+$ $\operatorname{Conv}\left(W_{K} \mu\right)$, whence $h(\nu) \leq h(\lambda)+h(\mu)$ by invariance and convexity of $\left.h\right)$. Moreover, $k[\mathcal{X}]$ is the Rees algebra associated with this filtration. It follows that

$$
k\left[\mathcal{X}_{0}\right]=k[\mathcal{X}] / z k[\mathcal{X}] \simeq \bigoplus_{\lambda \in \Lambda^{+} \cap \sigma} \text { End } V_{\lambda},
$$

as a $G \times G$-module, the multiplication being given by

$$
f_{\lambda} \cdot f_{\mu}=\sum_{\nu \in \Lambda^{+}, \nu \leq_{K} \lambda+\mu,\ulcorner h(\nu)\urcorner=\ulcorner h(\lambda)\urcorner+\ulcorner h(\mu)\urcorner} \operatorname{Tr}_{1}\left(p_{\lambda \mu}^{\nu}\left(f_{\lambda} \otimes_{k} f_{\mu}\right)\right)
$$

for any $f_{\lambda} \in$ End $V_{\lambda}$ and $f_{\mu} \in$ End $V_{\mu}$. Here for any $y \in \mathbb{R}$, we denote by $\ulcorner y\urcorner$ the largest integer $n$ such that $n \leq y$.

As a consequence, if there exists $\lambda \in \Lambda^{+} \cap \sigma$ such that $h(\lambda) \notin \mathbb{Z}$, then any $U^{-} \times U$-invariant function $f_{\lambda} \in$ End $V_{\lambda}$ is nilpotent; thus, $\mathcal{X}_{0}$ is not reduced. On the other hand, if $h\left(\Lambda^{+} \cap \sigma\right) \subseteq \mathbb{Z}$, then one has for any $U^{-} \times U$-invariant functions $f_{\lambda}, f_{\mu}$ :

$$
f_{\lambda} \cdot f_{\mu} \neq 0 \text { if and only if } h(\lambda)+h(\mu)=h(\lambda+\mu) .
$$

Since $h$ is positively homogeneous, it follows that the algebra $k\left[\mathcal{X}_{0}\right]^{U^{-} \times U}$ is reduced, so that $\mathcal{X}_{0}$ is reduced by Lemma 2.1. Moreover, its irreducible components are the $X_{\tau}$, where $\tau \subseteq \sigma$ is a maximal cone such that: $\left.h\right|_{\tau}$ is 
linear, and $\tau^{0}$ meets $\Lambda_{\mathbb{R}}^{+}$. Since $\mathcal{X}_{0}$ is a stable reductive variety (by Corollary (7.3), it follows easily that it is isomorphic to $X_{h}$.

Next we consider a family $\pi: \mathcal{X} \rightarrow S$ of stable reductive varieties over a nonsingular curve, that is, $S$ is integral, regular and of dimension 1 . We assume that the generic fiber of $\pi$ is geometrically irreducible; let $\sigma$ be the corresponding $W$-admissible cone. We show that $\pi$ looks locally like a family associated to a $W$-admissible height function on $\sigma$.

Proposition 7.13. With the preceding notation, for any closed point $s \in S$ there exist a nonsingular curve $S^{\prime}$, a closed point $s^{\prime} \in S^{\prime}$, two non-constant morphisms $\varphi:\left(S^{\prime}, s^{\prime}\right) \rightarrow(S, s)$ and $z:\left(S^{\prime}, s^{\prime}\right) \rightarrow\left(\mathbb{A}^{1}, 0\right)$, and a unique $W$ admissible height function $h: \sigma \rightarrow \mathbb{R}$, such that the pull-back families $\mathcal{X} \times{ }_{S} S^{\prime}$ and $\tilde{X}_{h} \times_{\mathbb{A}^{1}} S^{\prime}$ are isomorphic. In particular, the fiber $\mathcal{X}_{s}$ is isomorphic to $X_{h}$.

Proof. Using Corollary [7.9, we may assume that $\pi$ is trivial over the punctured curve $S-\{s\}$. We may further assume that $S$ is affine, regular, and admits a morphism $z: S \rightarrow \mathbb{A}^{1}$ having a unique zero at $s$, of order 1. Then $\mathcal{X}$ is affine. Setting $\mathcal{R}=\Gamma\left(\mathcal{X}, \mathcal{O}_{\mathcal{X}}\right)$ and $\mathcal{R}_{0}=\Gamma\left(S, \mathcal{O}_{S}\right)$, we have $\mathcal{R}_{0}[1 / z]=\Gamma\left(S-\{s\}, \mathcal{O}_{S}\right)$. Moreover, Lemma 7.2 yields an isomorphism of $\mathcal{R}_{0}-G \times G$-modules

embedded in

$$
\mathcal{R}=\bigoplus_{\lambda \in \Lambda^{+} \cap \sigma} F_{\lambda} \otimes_{k} \text { End } V_{\lambda}
$$

$$
\Gamma\left(\mathcal{X}-\mathcal{X}_{s}, \mathcal{O}_{\mathcal{X}}\right)=\mathcal{R}_{0}[1 / z] \otimes_{k} k\left[X_{\sigma}\right]=\bigoplus_{\lambda \in \Lambda^{+} \cap \sigma} \mathcal{R}_{0}[1 / z] \otimes_{k} \text { End } V_{\lambda}
$$

as a subalgebra. Here each $F_{\lambda}$ is an invertible $\mathcal{R}_{0}$-submodule of $\mathcal{R}_{0}[1 / z]$, trivial over $\mathcal{R}_{0}[1 / z]$. It follows that $F_{\lambda}=z^{h(\lambda)} \mathcal{R}_{0}$ for a unique map $h$ : $\Lambda^{+} \cap \sigma \rightarrow \mathbb{Z}$. We have for any $\lambda, \mu$ in $\Lambda^{+} \cap \sigma$ :

$$
z^{h(\lambda)} \text { End } V_{\lambda} \cdot z^{h(\mu)} \text { End } V_{\mu}=\bigoplus_{\nu \in \Lambda^{+}, \nu \leq K} z^{h(\lambda)+h(\mu)} \text { End } V_{\nu},
$$

where $K=K_{\sigma}$. It follows that $h(\nu) \leq h(\lambda)+h(\mu)$ for all such triples $\lambda, \mu$, $\nu$, and hence that the subspace

$$
R=\bigoplus_{\lambda \in \Lambda^{+} \cap C, n \in \mathbb{Z}, h(\lambda) \leq n} z^{n} \text { End } V_{\lambda} \subseteq k\left[X_{\sigma}\right]\left[z, z^{-1}\right] \subseteq \mathcal{R}
$$

is in fact a $G \times G$-invariant subalgebra containing $k[z]$.

Clearly, the natural map $R \otimes_{k[z]} \mathcal{R}_{0} \rightarrow \mathcal{R}$ is an algebra isomorphism. As a consequence, the map $R / z R \rightarrow k\left[\mathcal{X}_{s}\right]$ is an isomorphism; in particular, the algebra $R / z R$ is finitely generated, so that the same holds for $R^{U^{-} \times U} / z R^{U^{-} \times U}$. Now one easily checks that the algebra $R^{U^{-} \times U}$ is generated by $z$, together with any family of $T \times T$-eigenvectors which maps to generators of the quotient $R^{U^{-} \times U} / z R^{U^{-} \times U}$. It follows that $R$ is finitely generated. 
Let $\tilde{X}=\operatorname{Spec} R$. Then $\tilde{X}$ is equipped with a $G \times G$-action, and also with a compatible $\mathbb{G}_{m}$-action (since $R$ is graded by the degree in $z$ ). Thus, $\tilde{X}$ is a $\tilde{G} \times \tilde{G}$-variety, which is clearly irreducible, diagonal, and multiplicity-free, with weight set

$$
\left\{(\lambda, n) \mid \lambda \in \Lambda^{+} \cap \sigma, n \in \mathbb{Z}, h(\lambda) \leq n\right\} .
$$

We claim that this set is saturated in $\tilde{\Lambda}=\Lambda \times \mathbb{Z}$. Indeed, since $R / z R$ is reduced, one obtains $h(m \lambda)=m h(\lambda)$ for all $m \in \mathbb{Z}_{>0}$ and $\lambda \in \Lambda^{+} \cap \sigma$, by arguing as in the proof of Proposition 7.12. This implies the claim.

By that claim and Proposition 4.1, $\tilde{X}$ is a reductive variety for $\tilde{G}$. Now one easily checks that $\tilde{X}=\tilde{X}_{h}$ for a unique $W$-admissible height function $h: \sigma \rightarrow \mathbb{R}$ extending our original $h: \Lambda^{+} \cap \sigma \rightarrow \mathbb{Z}$.

We can now tackle the general case, when the generic fiber of a family is not geometrically irreducible. Let $\Sigma$ be a $W$-complex of cones.

Definition 7.14. A $W$-admissible system of heights on $\Sigma$ is a collection of height functions $\underline{h}=\left\{h_{\sigma}: \sigma \rightarrow \mathbb{R} \mid \sigma \in \Sigma\right\}$ such that for any $\sigma_{1}, \sigma_{2}, \tau$ in $\Sigma$ with $\tau \prec \sigma_{1} \cap \sigma_{2}$, the difference $\left.\left(h_{\sigma_{1}}-h_{\sigma_{2}}\right)\right|_{\tau}$ is a linear function.

Not let $t \in Z^{1}(\Sigma$, Aut) be a 1-cocycle defined over the field $k(z)$. We can write $t=z^{\gamma} t^{\prime}$, where $t^{\prime}$ is a 1-cocycle which is invertible at $z=0$, and $\gamma$ is obtained by taking the valuation of $t$ at 0 . Explicitly, $\gamma$ is a collection of homomorphisms $\gamma_{\tau} \in \operatorname{Hom}\left(\Lambda \cap \operatorname{lin} \tau / K_{\tau}, \mathbb{Z}\right)$ for all $\tau \prec \sigma_{1} \cap \sigma_{2}$ (that is, of one-parameter subgroups of Aut ${ }^{G \times G}\left(X_{\tau}\right)$ ) which satisfy the 1-cocycle condition on all triple intersections. We call $t$ admissible if the following condition holds for all faces $\tau \prec \sigma_{1} \cap \sigma_{2}$ :

$$
\left.\left(h_{\sigma_{1}}-h_{\sigma_{2}}\right)\right|_{\tau}=\gamma_{\tau} \text {. }
$$

Then we can define a family $\tilde{X}_{\underline{h}, t}$ over the open subset of $\mathbb{A}^{1}$ (containing 0 ) where $t^{\prime}$ is regular. It will be a family of stable reductive varieties precisely when all $h_{\sigma}$ are $\mathbb{Z}$-valued.

Indeed, each height function $h_{\sigma}$ defines a subdivision of the cone $\sigma$ and a family $\tilde{X}_{h_{\sigma}}$ corresponding to a subalgebra of $k[G]\left[z, z^{-1}\right]$. We then just glue them along $t$. It is clear that the general fiber of $\tilde{X}_{\underline{h}, t}$ is the union of varieties $X_{h_{\sigma}}$ glued along $t^{\prime}(0)$. Vice versa, one has

Proposition 7.15. Let $\pi: \mathcal{X} \rightarrow S$ be a family of stable reductive varieties over a nonsingular curve germ $(S, s)$, and assume that the generic fiber is multiplicity-free. Then after a nonconstant base change $\left(S^{\prime}, s^{\prime}\right) \rightarrow(S, s)$ the family $\mathcal{X}^{\prime}$ is a pullback of some $\tilde{X}_{\underline{h}, t}$ under $\left(S^{\prime}, s^{\prime}\right) \rightarrow\left(\mathbb{A}^{1}, 0\right)$.

Proof. After making a finite base change, we can assume that every irreducible component $\mathcal{Y}_{\eta}$ of the generic fiber $\mathcal{X}_{\eta}$ is geometrically irreducible and is trivial over $k(\eta)$. Its closure $\overline{\mathcal{Y}_{\eta}}=\mathcal{Y}$ in $\mathcal{X}$ is $G \times G$-invariant and reduced, so must be flat over $S$. Thus, $\mathcal{Y}$ is a family of stable reductive varieties, with geometrically irreducible generic fiber. By Proposition 7.13 we may assume that $\mathcal{Y}$ is a pull-back of $\tilde{X}_{h_{\sigma}}$, where $h_{\sigma}$ is a well-defined weight 
function. This defines a $W$-complex of cones with an injective reference map, since $\mathcal{X}$ is multiplicity-free. Any intersection $\tau=\sigma_{1} \cap \sigma_{2}$ is a cone, and $\tilde{X}_{h_{\tau}}$ embeds into both $\tilde{X}_{h_{\sigma_{1}}}$ and $\tilde{X}_{h_{\sigma_{2}}}$. These identifications define an admissible cocycle $t$.

7.5. The Vinberg family. We construct a family of $G \times G$-varieties with general fiber $G$, after Vinberg Vin95]. Consider the algebra

$$
k[T \times G]=\bigoplus_{\lambda \in \Lambda^{+}, \mu \in \Lambda} e^{\mu} \text { End } V_{\lambda}
$$

(where $e^{\mu}$ denotes the weight $\mu$ regarded in $k[T]$ ), and its subspace

$$
R=\bigoplus_{\lambda \in \Lambda^{+}, \mu \in \Lambda, \lambda \leq \Pi \mu} e^{\mu} \text { End } V_{\lambda} .
$$

Then $R$ is invariant under the product action of $T \times G \times G$, and is also a subring of $k[T \times G]$, since

$$
\text { End } V_{\lambda} \cdot \text { End } V_{\mu} \subseteq \bigoplus_{\nu \leq \Pi \lambda+\mu} \text { End } V_{\nu}
$$

for all $\lambda, \mu \in \Lambda^{+}$. Note that the invariant subalgebra

$$
R_{0}=R^{G \times G} \simeq \bigoplus_{\mu \in \Lambda, \mu \geq \Pi_{\Pi} 0} k e^{\mu}
$$

is the polynomial ring $k\left[e^{\alpha}, \alpha \in \Pi\right]$, that is, the coordinate ring of the affine space $\mathbb{A}^{\Pi}$. Moreover, the subalgebra

$$
R^{U^{-} \times U}=\bigoplus_{\lambda \in \Lambda^{+}, \mu \in \Lambda} e^{\mu}\left(\text { End } V_{\lambda}\right)^{U^{-} \times U}
$$

is isomorphic to $k\left[\Lambda^{+}\right] \otimes_{k} R_{0}$, where $k\left[\Lambda^{+}\right]=k[G]^{U^{-} \times U}$ is the algebra of the monoid of dominant weights. In particular, the algebra $R^{U^{-} \times U}$ is a finitely generated normal domain; thus, the same holds for $R$ by Gro97. Theorem 16.2. So $\mathcal{V}=\operatorname{Spec} R$ is a $T \times G \times G$-variety, endowed with a morphism

$$
p: \mathcal{V} \rightarrow \mathbb{A}^{\Pi}
$$

that is $G \times G$-invariant and $T$-equivariant (where $T$ acts linearly on $\mathbb{A}^{\Pi}$ with weights the simple roots). One has an isomorphism of $R_{0}$-modules

$$
R \simeq \bigoplus_{\lambda \in \Lambda^{+}} R_{0} \otimes_{k} e^{\lambda} \text { End } V_{\lambda}
$$

It follows that $p$ is flat, and that $p / /\left(U^{-} \times U\right): \mathcal{V} / /\left(U^{-} \times U\right) \rightarrow \mathbb{A}^{\Pi}$ is a trivial family of $T$-varieties; its fiber is the affine toric variety associated with the monoid $\Lambda^{+}$. Thus, by Lemma 2.1. all fibers of $p$ are reduced and irreducible.

Lemma 7.16. (1) The map $p: \mathcal{V} \rightarrow \mathbb{A}^{\Pi}$ is a family of reductive varieties for $G$, the Vinberg family. 
(2) The fiber of $p$ at any closed point of $\mathbb{A}^{\Pi}$ with non-zero coordinates is isomorphic to $G$.

(3) Let $X$ be an affine $G$-variety and let

$$
p_{X}: X * \mathcal{V}=(X \times \mathcal{V}) / / G \rightarrow \mathcal{V} / / G=\mathbb{A}^{\Pi}
$$

be the family of $G$-varieties defined in Lemma 7.5. Then the induced map $p_{X} / / U:(X * \mathcal{V}) / / U \rightarrow \mathbb{A}^{\Pi}$ is a trivial family of $T$-varieties. As a consequence, if $X$ is irreducible, then so are all fibers of $p_{X}$. Moreover, the fiber of $p_{X}$ at any closed point with non-zero coordinates is isomorphic to $X$.

Proof. (1) has just been proved.

(2) Note that the complement in $\mathbb{A}^{\Pi}$ of the union of all coordinate hyperplanes is a torus with character group the root lattice. So this torus is the quotient of $T$ by the center $Z(G)$. Moreover, the pull-back of $p$ to this torus is the natural map $(T \times G) / Z(G) \rightarrow T / Z(G)$, where $Z(G)$ acts on $T \times G$ by $z(t, g)=(z t, z g)$. Thus, its fibers are isomorphic to $G$.

(3) Since $X * \mathcal{V}=(X \times \mathcal{V}) / / G$, one has

$$
k[X * \mathcal{V}]^{U}=\bigoplus_{\lambda \in \Lambda^{+}, \mu \in \Lambda, \lambda \leq_{\Pi} \mu} e^{\mu} k[X]_{\lambda}^{U},
$$

a subalgebra of $k[T \times X]^{U}$. Thus, the $T$-invariant subspace

$$
A=\bigoplus_{\lambda \in \Lambda^{+}} e^{\lambda} k[X]_{\lambda}^{U}
$$

is a subalgebra of $k[X * \mathcal{V}]^{U}$, isomorphic to $k[X]^{U}$. Moreover, $k[X * \mathcal{V}]^{U}$ is isomorphic to $A \otimes_{k} R_{0}$ as a $T$-algebra. This trivializes $p_{X} / / U$, and proves the first assertion. Together with Lemma 2.1 this implies the second assertion.

Next we describe all fibers of $p_{X}$; for this, we recall a construction that already occurred in the proof of Theorem [7.6 Let $\gamma$ be a dominant oneparameter subgroup of $T$. For any non-negative integer $n$, let

$$
k[X]_{\leq n}=\bigoplus_{\lambda \in \Lambda^{+},\langle\lambda, \gamma\rangle \leq n} \operatorname{Hom}^{G}\left(V_{\lambda}, k[X]\right) \otimes_{k} V_{\lambda}
$$

(where we identify each $\operatorname{Hom}^{G}\left(V_{\lambda}, k[X]\right) \otimes_{k} V_{\lambda}$ with its image in $k[X]$ via the evaluation map). Then the $k[X]_{\leq n}$ form an ascending filtration of the ring $k[X]$ by $G$-submodules. The associated graded $\operatorname{ring} \operatorname{gr}_{\gamma} k[X]$ is isomorphic (as a $G$-module) to $k[X]$; we may regard the product $V_{\lambda} \cdot V_{\mu}$ of any two simple submodules of $\operatorname{gr}_{\gamma} k[X]$ as the subspace of their product in $k[X]$ spanned by those $V_{\nu}$ such that $\langle\gamma, \nu\rangle=\langle\gamma, \lambda+\mu\rangle$. Equivalently, $\nu \leq_{K} \lambda+\mu$, where $K$ is the set of simple roots orthogonal to $\gamma$; in particular, $\operatorname{gr}_{\gamma} k[X]$ depends only on $K$. For example, $\operatorname{gr}_{\gamma} k[G]$ is the algebra $k[G]_{(K)}$ considered in Subsection 4.1 .

We can now state the following result, whose proof is a direct checking. 
Lemma 7.17. Given a closed point $s=\left(s_{\alpha}\right)_{\alpha \in \Pi} \in \mathbb{A}^{\Pi}$, let $K=\{\alpha \in$ $\left.\Pi \mid s_{\alpha}=0\right\}$ and let $\gamma$ be a dominant one-parameter subgroup of $T$, such that $K=\{\alpha \in \Pi \mid\langle\gamma, \alpha\rangle=0\}$. Then the algebra of regular functions on the fiber of $p_{X}$ at $s$ is isomorphic to $\mathrm{gr}_{\gamma} k[X]$.

In particular, the fiber of the Vinberg family at $s$ is the reductive variety $X_{\Lambda_{\mathbb{R}}^{+}, K}=\operatorname{Spec} k[G]_{(K)}=\operatorname{Spec} k\left[G \times G / H_{K, \Lambda, \emptyset}\right]$.

If $s$ lies outside all coordinate hyperplanes of $\mathbb{A}^{\Pi}$, then $K=\emptyset$. Then we can take $\gamma=0$, whence $(X * \mathcal{V})_{s} \simeq X$ (this also follows from Lemma [7.5). On the other hand, if $s$ is the origin of $\mathbb{A}^{\Pi}$, then $K=\Pi$, the dominant one-parameter subgroup $\gamma$ is regular, and $\operatorname{gr}_{\gamma} k[X]$ is the degeneration of $k[X]$ constructed in Pop86 (see also Gro97] Chapter 15).

7.6. Local structure of families of reductive varieties. We show that the Vinberg family is a local model (in the sense of Proposition 7.13) for families of reductive varieties.

Theorem 7.18. Let $\pi: \mathcal{X} \rightarrow S$ be a family of reductive varieties over an integral scheme, with general fiber $X=X_{\sigma}$; let $K=K_{\sigma}$. Then every $s \in S$ admits an open neighborhood $S^{\prime}$ and a morphism $f: S^{\prime} \rightarrow \mathbb{A}^{K}$, such that the pull-back families $\mathcal{X} \times{ }_{S} S^{\prime}$ and $(X * \mathcal{V}) \times_{\mathbb{A} \Pi} S^{\prime}$ are isomorphic (here we regard $\mathbb{A}^{K}$ as a coordinate subspace of $\left.\mathbb{A}^{\Pi}\right)$.

Proof. We may assume that $S$, and hence $\mathcal{X}$, is affine, and also (by Lemma (7.4) that the family $\pi / /\left(U^{-} \times U\right): \mathcal{X} / /\left(U^{-} \times U\right) \rightarrow S$ is trivial. Let $\mathcal{R}=\Gamma\left(\mathcal{X}, \mathcal{O}_{\mathcal{X}}\right)$ and $\mathcal{R}_{0}=\Gamma\left(S, \mathcal{O}_{S}\right)$. As in the proof of Corollary [7.9, write

$$
\mathcal{R}=\bigoplus_{\lambda \in \Lambda^{+} \cap \sigma} F_{\lambda} \otimes_{k} \text { End } V_{\lambda} \subseteq \bigoplus_{\lambda \in \Lambda^{+} \cap \sigma} k(\bar{S}) \otimes_{k} \text { End } V_{\lambda},
$$

where every $F_{\lambda}$ is an invertible $\mathcal{R}_{0}$-submodule of $k(\bar{S})$. Then we may choose local generators $f_{\lambda}$ of $F_{\lambda}$ at $s$, satisfying $f_{\lambda} f_{\mu}=f_{\lambda+\mu}$ for all $\lambda, \mu$ in $\Lambda^{+} \cap \sigma$.

Since the group $\Lambda \cap \operatorname{lin}(\sigma)$ is generated by $\Lambda^{+} \cap \sigma$, the assignment $\lambda \mapsto f_{\lambda}$ extends uniquely to a group homomorphism $\Lambda \cap \operatorname{lin}(\sigma) \rightarrow k(\bar{S})^{*}$. And since $K$ is contained in $\Lambda \cap \operatorname{lin}(\sigma)$, we obtain in particular elements $f_{\alpha} \in k(\bar{S})^{*}$ $(\alpha \in K)$.

Let $\alpha \in K$. By Lemma 3.5 we may find $\lambda \in \Lambda^{+} \cap \sigma$ such that $\langle\lambda, \check{\alpha}\rangle \neq 0$; then the product $f_{\lambda}$ End $V_{\lambda} \cdot f_{\lambda}$ End $V_{\lambda}$ contains $f_{\lambda}^{2}$ End $V_{2 \lambda-\alpha}$. It follows that

$$
f_{2 \lambda}=f_{\lambda}^{2} \in F_{2 \lambda-\alpha}=f_{2 \lambda-\alpha} \mathcal{R}_{0}=f_{2 \lambda} f_{\alpha}^{-1} \mathcal{R}_{0},
$$

that is, $f_{\alpha} \in \mathcal{R}_{0}$. Thus, we obtain a morphism $f=\left(f_{\alpha}\right)_{\alpha \in K}: S \rightarrow \mathbb{A}^{K}$.

Let $R_{0}$ be the subalgebra of $\mathcal{R}_{0}$ generated by the $f_{\alpha}, \alpha \in K$. We claim that the subspace of $\mathcal{R}$ :

$$
R=\bigoplus_{\lambda \in \Lambda^{+} \cap \sigma, \mu \in \Lambda, \lambda \leq K_{K} \mu} k f_{\mu} \otimes_{k} \text { End } V_{\lambda}=\bigoplus_{\lambda \in \Lambda^{+} \cap \sigma} f_{\lambda} R_{0} \otimes_{k} \text { End } V_{\lambda}
$$

is a subalgebra over $R_{0}$. Indeed, if $\lambda, \mu \in \Lambda^{+} \cap \sigma$ and $\nu \in \Lambda^{+}$satisfies $\nu \leq_{K} \lambda+\mu$, then $f_{\lambda} f_{\mu}=f_{\nu} f_{\lambda+\mu-\nu} \in f_{\nu} R_{0}$. 
Clearly, the multiplication map

$$
R \otimes_{R_{0}} \mathcal{R}_{0} \rightarrow \mathcal{R}
$$

is an isomorphism of $\mathcal{R}_{0}$-algebras. In other words, $\mathcal{X}$ is the pull-back of the family $p_{X}: X * \mathcal{V} \rightarrow \mathbb{A}^{\Pi}$ under $f$.

\section{REFERENCES}

[Ale02] V. Alexeev, Complete moduli in the presence of semiabelian group action, Ann. of Math., 155 (2002), no. 3, 611-708.

[SRV-II] V. Alexeev and M. Brion, Stable reductive varieties II: Projective case, preprint, arXiv:math.AG/0207274 to appear in Adv. Math.

[DM69] P. Deligne and D. Mumford, The irreducibility of the moduli space of curves of given genus, Inst. Hautes Études Sci. Publ. Math. 36 (1969), 75-110.

[Ful93] W. Fulton, Introduction to Toric Varieties, Annals of Mathematics Studies 131, Princeton University Press, 1993.

[Gro97] F. Grosshans, Algebraic homogeneous spaces and invariant theory, Lecture Note in Mathematics 1673, Springer-Verlag, 1997.

[Har77] R. Hartshorne, Algebraic geometry, Graduate Texts in Mathematics 52, Springer-Verlag, 1977.

[HS02] M. Haiman and B. Sturmfels, Multigraded Hilbert schemes, preprint, arXiv:math.AG/0201271, to appear in J. Algebraic Geom.

[Kno89] F. Knop, The Luna-Vust Theory of Spherical Embeddings, in: Proceedings of the Hyderabad Conference on Algebraic Groups, 225-249, Manoj-Prakashan, 1989

[Kno94] ㄴ The asymptotic behavior of invariant collective motion, Invent. math. 116 (1994), 309-328.

[Lun75] D. Luna, Adhérences d'orbites et invariants, Invent. math. 29 (1975), 231238.

[Mum94] D. Mumford, Geometric invariant theory, third enlarged ed., Ergebnisse der Mathematik und ihrer Grenzgebiete 34, Springer-Verlag, 1994.

[Nam76] Y. Namikawa, A new compactification of the Siegel space and degenerations of abelian varieties, I, II., Math. Ann. 221 (1976), 97-141, 201-241.

[Pop86] V. L. Popov, Contractions of actions of reductive algebraic groups, Mat. Sb. (N.S.) 130 (1986), 310-334.

[PopVin94] V. L. Popov and E. B. Vinberg, Invariant theory, Encyclopaedia of Mathematical Sciences 55, Springer-Verlag, Berlin, 1994.

[Put82] M. S. Putcha, Green's relations on a connected algebraic monoid, Linear and Multilinear Algebra 12 (1982/83), 205-214.

[Put84] Reductive groups and regular semigroups, Semigroup Forum 30 (1984), 253-261.

[PutRen88] M. S. Putcha and L. E. Renner, The system of idempotents and the lattice of $\mathcal{J}$-classes of reductive algebraic monoids, J. Algebra 116 (1988), 385-399.

[Rit98] A. Rittatore, Algebraic monoids and group embeddings, Transf. Groups 3 (1998), 375-396.

[Sol99] L. Solomon, An introduction to reductive monoids, in: Semigroups, formal languages and groups (York, 1993), 295-352, NATO Adv. Sci. Inst. Ser. C Math. Phys. Sci., 466, Kluwer Acad. Publ., Dordrecht, 1995.

[Vin86] E. B. Vinberg, Complexity of actions of reductive groups, Functional Anal. Appl. 20 (1986), 1-11.

[Vin95] _ On reductive algebraic semigroups, in: Lie groups and Lie algebras: E. B. Dynkin's Seminar, 145-182, Amer. Math. Soc. Transl. Ser. 2, 169, 1995. 
Department of Mathematics, University of Georgia, Athens, GA 30602, USA

E-mail address: valery@math.uga.edu

Institut Fourier, B. P. 74, 38402 Saint-Martin d'Hères Cedex, France

E-mail address: Michel.Brion@ujf-grenoble.fr 